\title{
SPECTRA OF HIGH-IONIZATION SEYFERT 1 GALAXIES: IMPLICATIONS FOR THE NARROW-LINE REGION \\ David MoOre, ${ }^{1}$ Ross D. COHEN, ${ }^{1}$ AND Geoffrey W. MARCY ${ }^{2}$ \\ Received 1995 February 22; accepted 1996 April 23
}

\section{ABSTRACT}

We present line profiles and profile parameters for the narrow-line regions (NLRs) of six Seyfert 1 galaxies with high-ionization lines: MCG 8-11-11, Mrk 79, Mrk 704, Mrk 841, NGC 4151, and NGC 5548. The sample was chosen primarily with the goal of obtaining high-quality [Fe vII] $\lambda 6087$ and, when possible, $[\mathrm{Fe} \mathrm{x}] \lambda 6374$ profiles to determine if these lines are more likely formed in a physically distinct "coronal line region" or are formed throughout the NLR along with lines of lower critical density $\left(n_{\mathrm{cr}}\right)$ and/or ionization potential (IP).

We discuss correlations of velocity shift and width with $n_{\mathrm{cr}}$ and IP. In some objects, lines of high IP and/or $n_{\mathrm{cr}}$ are systematically broader than those of low IP $/ n_{\mathrm{cr}}$. Of particular interest, however, are objects that show no correlations of line width with either IP or $n_{\mathrm{cr}}$. In these objects, lines of high and low IP $/ n_{\mathrm{cr}}$ are remarkably similar, which is difficult to reconcile with the classical picture of the NLR, in which lines of high and low IP $/ n_{\mathrm{cr}}$ are formed in physically distinct regions. We argue for similar spatial extents for the flux in lines with similar profiles.

Here, as well as in a modeling-oriented companion paper (Paper II), we develop further an idea suggested by Moore \& Cohen that objects that do and do not show line width correlations with $\mathrm{IP} / n_{\mathrm{cr}}$ can both be explained in terms of a single NLR model with only a small difference in the cloud column density distinguishing the two types of object. Overall, our objects do not show correlations between the full width at half-maximum (FWHM) and IP and/or $n_{\mathrm{cr}}$. The width must be defined by a parameter that is sensitive to extended profile wings in order for the correlations to result. In Paper II, we present models in which FWHM correlations with IP and/or $n_{\mathrm{cr}}$ result only after simulating the lower spectral resolution used in previous observational studies. The models that simulate the higher spectral resolution of our observational study produce line width correlations only if the width is defined by a parameter that is more sensitive to extended profile wings than is the FWHM. Our sample of six objects is in effect augmented by incorporating the larger sample ( 16 objects) of Veilleux into some of our discussion.

This paper focuses on new interpretations of NLR emission-line spectra and line profiles that stem directly from the observations. Paper II focuses on modeling and complements this paper by illustrating explicitly the effects that spatial variations in electron density, ionization parameter, and column density have on model profiles. By comparing model profiles with the observed profiles presented here, as well as with those presented by Veilleux, Paper II yields insight into how the electron density, ionization parameter, and column density likely vary throughout the NLR.

Subject headings: galaxies: nuclei — galaxies: Seyfert — line: profiles

\section{INTRODUCTION}

One well-known observational feature of the narrow-line region (NLR) in Seyfert galaxies is the preponderance of blueward asymmetries in the emission-line profiles (e.g., Heckman et al. 1981; Whittle 1985a; Dahari \& De Robertis 1988; De Robertis and Shaw 1990; Veilleux 1991a). To account for the asymmetry, it is accepted in general that the NLR velocity field is mostly radial and that some source of opacity, most likely dust, is present. However, a consensus regarding the direction of cloud flow has yet to emerge because the blueward asymmetry can be explained qualitatively by either internal obscuration in the case of inflow or by external obscuration in the case of outflow (e.g., Capriotti, Foltz, \& Byard 1981).

Another well-known observational feature of the NLR is that the full width at half-maximum (FWHM) of emission lines often correlate with ionization potential (IP) and critical density $\left(n_{\mathrm{cr}}\right)$. This feature has been commonly used to argue for a velocity field increasing radially inward under

\footnotetext{
${ }^{1}$ Center for Astrophysics and Space Sciences 0111, 9500 Gilman Drive, University of California, San Diego, La Jolla, CA 92093-0111.

2 Department of Astronomy, University of California, Berkeley, Berkeley, CA 94720 .
}

the assumption that lines of low $\mathrm{IP} / n_{\mathrm{cr}}$ are emitted less efficiently (for fixed emitting mass) close to the central source than lines of high IP/ $n_{\mathrm{cr}}$ (e.g., Osterbrock 1981; De Robertis \& Osterbrock 1984, 1986, Whittle 1985b; Appenzeller \& Östreicher 1988; De Robertis \& Shaw 1990; Veilleux 1991b).

By comparing the FWHM of [O I] $\lambda 6300$ to that of [S II] $\lambda 6716$ in Seyfert galaxies, and by comparing the FWHM of [O $\mathrm{III}] \lambda 4363$ to that of $[\mathrm{O} \mathrm{III}] \lambda 5007$ in Seyfert 2 galaxies (in which there is little or no contamination from broad $\mathrm{H} \gamma$ and/or Fe II emission), we have argued that the correlations are best interpreted as fundamentally with ionization potential rather than with critical density (Moore \& Cohen 1994, hereafter MC). We also proposed a new interpretation that these correlations are a column density effect, in which case the correlations are also consistent both with constant ionization parameter and with velocity increasing outward. As we pointed out in MC, line width is correlated with neither critical density nor ionization potential in some objects. In these objects, the similarity between profiles spanning a broad range of ionization potential and critical density is inconsistent with a scenario in which lines of low and high $\mathrm{IP} / n_{\mathrm{er}}$ are formed in physically distinct regions. The [Fe vII] 
$=$

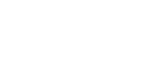

. 
$\lambda 6087$ and $[\mathrm{Fe} \mathrm{x}] \lambda 6374$ lines, which are very sensitive to ionization parameter (MC), are particularly important in this regard, and we emphasize these lines here.

In $\S 3$, we present a sample of narrow-line profiles in intermediate Seyfert galaxies. The sample was chosen with the goal of obtaining high-quality [Fe vII] $\lambda .6087$ and, when possible, $[\mathrm{Fe} \mathrm{x}] \lambda 6374$ profiles. The sample consists of MCG 8-11-11, Mrk 79, Mrk 704, Mrk 841, NGC 4151, and NGC 5548.

We discuss briefly details of the observations and of the data reduction and profile extraction in $\S 2$. In $\S 3$, we discuss general implications for the NLR of line profile features. This discussion is based on previous observational studies as well as on our own. It will be useful to have already highlighted those general features of NLR spectra that discriminate best between different NLR models when we discuss our spectra and profiles. In $\S 4$ we discuss specific implications of our data for NLR models. This discussion is facilitated by references to models presented in a companion paper (Moore \& Cohen 1996, hereafter Paper II). The most constraining feature of the observed line profiles is the similarity in some objects of the profiles of lines corresponding to a wide range of IP $/ n_{\mathrm{cr}}$, and with very different sensitivities to ionization parameter. In $\S 5$, we confirm for our sample the well-known correlations of velocity shift and width with IP and $n_{\mathrm{cr}}$. However, our sample, which includes one contradictory object (NGC 5548), is too small to confirm by itself the conclusion of MC that the correlations are fundamentally with ionization potential rather than with critical density. Finally, we summarize our results in $\S 6$.

\section{DATA ANALYSIS}

\subsection{Observations and Reductions}

Observations were made with the 200 inch $(5.08 \mathrm{~m})$ Hale telescope and double spectrograph (Oke \& Gunn 1982) on six nights in 1983 and on a seventh night in 1984. The double spectrograph incorporates a dichroic beam splitter to cover approximately $3100-5200 \AA$ on the blue side and $5200-11000 \AA$ on the red side. The blue side had an RCA $320 \times 512 \mathrm{CCD}$ with $30 \mu \mathrm{m}$ pixels, which corresponds to a spatial scale of $0^{\prime \prime} 8$ pixel $^{-1}$. One DN (count) corresponds to 6 photoelectrons, and the readout noise was $8 \mathrm{DN}$ pixel $^{-1}$. A $1200 \mathrm{~g} \mathrm{~mm}^{-1}$ grating blazed at $4700 \AA$ yields a dispersion of $1.09 \AA$ pixel $^{-1}$ at $3500 \AA$ ( $200 \mathrm{~km} \mathrm{~s}^{-1}$ FWHM). The red side had a TI $800 \times 800 \mathrm{CD}$ with $15 \mu \mathrm{m}$ pixels, which corresponds to a spatial scale of $0 " 6$ pixel $^{-1}$. One DN corresponds to 2 photoelectrons, and the readout noise was 5 DN pixel ${ }^{-1}$. A $1200 \mathrm{~g} \mathrm{~mm}^{-1}$ grating blazed at $7100 \AA$ yields a dispersion of $0.82 \AA$ pixel $^{-1}$ at $6300 \AA\left(75 \mathrm{~km} \mathrm{~s}^{-1}\right.$ FWHM).

Because of the small wavelength coverage, most observations required unique wavelength settings. Grating tilts were set by hand with a vernier reading, which could not be reset to a specified wavelength with high precision. For most observations, wavelength calibration lamps and flatfield exposures were taken immediately before or after the observation at the hour angle and declination of the start or end of the observation, in order to minimize errors due to flexure in the spectrograph. Occasionally, calibration exposures were taken between two identical setups on an object. All observations were made with a 1 " slit except one ( $\lambda 4363$ in NGC 5548).
For all the data reduction, we used the astronomical data reduction software package VISTA, developed at Lick Observatory. Straightforward procedures were used for baseline and LED preflash subtraction (for exposures in the blue) as well as for flat-fielding.

For each column, the spatial profile of the sky background was modeled by a fourth-order polynomial fit outside those rows bracketing the spatial profile of the object. The procedure includes automatic detection and rejection of cosmic-ray events as well as of hot and cold pixels in the sky background. From each column of the exposure, we subtracted the sky background model for that column.

The orientation of the $\mathrm{CCD}$ was skewed slightly with respect to the grating rulings, resulting in a shift in the centroid of the spatial profile of about 3 pixels over the length of the spectrum. The spectrum was extracted from a symmetric window, typically $6-8$ pixels wide, centered on a parabolic fit to the centroid curve. The extraction included interpolation to allow for fractional pixels.

The size of the window used for spectral extractions was chosen to be roughly the apparent full width at zerointensity of the spatial profile of the nuclear continuum. Observations were acquired under a range of atmospheric seeing conditions as follows. Spectra of MCG 8-11-11 were acquired under $3^{\prime \prime}-4^{\prime \prime}$ seeing. Spectra of Mrk 79 were acquired under 1".5 seeing. Separate spectra of Mrk 704 were acquired under $1^{\prime \prime}, 11^{\prime \prime} 5,2^{\prime \prime}$, and $4^{\prime \prime}-5^{\prime \prime}$ seeing. Spectra of Mrk 841 were acquired under 1.5 and $3^{\prime \prime}$ seeing. Separate spectra, covering the same wavelength region, of NGC 4151 were acquired under 1".5 (Fig. 5a), and 4"-5" (Fig. 5b) seeing. Spectra of NGC 5548 were acquired under 1 ".5-2" seeing. Even for NGC 4151, the nearest Seyfert galaxy in the sample, and for which separate spectra were acquired under the most extreme differences in seeing, spectra obtained under different seeing conditions showed very small, if any, systematic differences.

The extracted spectra were wavelength calibrated using polynomial fits to the measured centroids and known wavelengths of approximately 10 comparison lines in the blue and approximately 15 lines in the red. The order of the polynomial ranged between 2 and 4 and resulted in an rms residual of $0.02-0.06 \AA$. The absolute accuracy of the wavelength calibration can be inferred only from the night-sky lines in the spectrum. Offsets between the wavelengthcalibrated object spectra and those of the night sky were of the order of $0.2 \AA$ in scans with night-sky lines strong enough to permit a reliable comparison. The absolute accuracy could be better than $0.2 \AA$ because this is roughly the precision with which the centroids of night-sky lines can be calculated. After extracting each spectrum and correcting for atmospheric extinction, the spectrum of a standard star was used to flux calibrate the object spectrum.

Occasionally an emission line of interest is close in wavelength to an atmospheric absorption feature. To correct for the feature, we used a transmission curve derived from a normalized standard star spectrum, in which the depth of the absorption was determined empirically by comparing the depths of the absorption troughs in the object and standard star (which were observed at slightly different air mass).

There were two profiles that were seriously corrupted by atmospheric absorption, those of [S $\mathrm{II}$ ] $\lambda 6731$ in MCG 8-1111 and of $[S \mathrm{II}] \lambda 6716$ in Mrk 79 (both by $B$ band). We have 
excluded these profiles from formal presentation and analysis. However, profile parameters were calculated nonetheless, and after correcting for the absorption, the parameters calculated for $\lambda 6716$ in MCG 8-11-11 were in reasonable agreement with those calculated for $\lambda 6731$. Similarly, the parameters of $\lambda 6731$ in Mrk 79 agreed with those of $\lambda 6716$. In Mrk 704, the $B$-band feature ends just blueward of [S II] 26716 , and inspection of the standard star shows that the atmospheric absorption does not affect the emission line directly. Nevertheless, the absorption complicated selection of the blue wing of the $\lambda 6716$ profile enough that, after subtracting off an estimated underlying red wing of $\mathrm{H} \alpha$ broad, we considered the profile unsuitable for further analysis. The atmospheric absorption feature at $6280 \AA$ is just redward of the $[\mathrm{Fe}$ vII $\lambda 6087$ profile in Mrk 704, but again inspection of the standard star spectrum shows that the line profile is not affected directly. The $[\mathrm{Fe} \mathrm{VII}]$ line of Mrk 704 was not excluded from further analysis because the strength and shape of the adjacent continuum permitted reliable subtraction, despite the absorption feature. The $[\mathrm{Ne}$ III] $\lambda 3869$ profile in MCG 8-11-11 is just blueward of Ca II absorption at $3935 \AA$ from the Galaxy, but the absorption feature is narrow, and again neither the profile nor the continuum subtraction are affected seriously.

Several of the emission lines were observed in more than one scan either because several observations were made with the same grating tilt or because spectra overlapped enough in wavelength to include a common emission line. Such scans were averaged weighting by $(\mathrm{S} / \mathrm{N})^{2}$, where the signal-to-noise ratio $(\mathrm{S} / \mathrm{N})$ in each spectrum was determined empirically from the rms scatter about a low-order polynomial fit to a featureless part of the continuum.

\subsection{Profile Extraction}

We chose the limits of each emission line by visual inspection and subtracted a polynomial fit to a featureless part of the adjacent continuum. The profiles were then placed on a velocity scale. Small heliocentric corrections were applied to the velocities as appropriate. In the ideal case, the zero point of the velocity scale corresponds to the recessional velocity of the NLR center of mass. The least subjective estimate of the NLR center-of-mass velocity is that of $21 \mathrm{~cm}$ radiation. We used a $21 \mathrm{~cm}$ measurement, taken from Heckman, Balick, \& Sullivan (1978), for Mrk 79, NGC 4151, and NGC 5548. A $21 \mathrm{~cm}$ measurement was not available for the remaining objects. We used the peak of [O III] $\lambda 5007$ for Mrk 704 , the centroid of [O I] $\lambda 6300$ for MCG $8-11-11$, and the peak of [O 1$] 26300$ for Mrk 841 . The appropriate measurement was picked from observational considerations such as the $\mathrm{S} / \mathrm{N}$, profile asymmetry, and/or the degree to which a profile peak could be determined unambiguously, rather than from model-dependent considerations in which a particular measurement is assumed a priori to best represent the galactic systemic velocity.

In some cases, the spread in cloud velocity is large enough to blend together the individual profiles of two nearby emission lines. To reconstruct the individual profiles, we used a deblending program developed at UCSD by Steve Bradley. The program allows a template profile to be defined from the data, from which model profiles can be derived by rescaling the template in intensity and by using interpolated profile fluxes to vary the wavelength and width of the template.

Further details of the appropriate deblending procedure depend on the nature of the blend. We describe the deblending of four particularly important lines, [O III] $\lambda 4363$ (highdensity diagnostic) from broad and narrow $\mathrm{H} \gamma,[\mathrm{Fe} \mathrm{X}]$ $\lambda 6374$ (high-ionization parameter diagnostic) from [O I] $\lambda 6363,[\mathrm{O} \mathrm{I}] \lambda 6300$ (large column density diagnostic) from $\left[\begin{array}{ll}\mathrm{S} & \mathrm{III}\end{array}\right] \lambda 6312$, and $[\mathrm{S}$ II] $\lambda \lambda 6716,6731$ (low-density diagnostic).

To subtract $\mathrm{H} \gamma$ from [O III] $\lambda 4363$, we used the broad and narrow components of $\mathrm{H} \beta$ to model the respective components of $\mathrm{H} \gamma$, scaling them in intensity and width and varying the position until the final profile matched, in a least-squares sense, that portion of the $\mathrm{H} \gamma$ profile not contaminated by [O III] $\lambda 4363$ emission. We did not assume that the ratios of the narrow and broad components are the same for both $\mathrm{H} \gamma$ and $\mathrm{H} \beta$. For Mrk 79 and NGC 5548, an analogous procedure was used to deblend [S III] $\lambda 6312$ from [O I] $\lambda 6300$ using [O III] $\lambda 5007$ as a template [O I] profile. The net result here is to replace that portion of the [O I] profile contaminated by [S III] emission by a template [O III] $\lambda 5007$ profile, where the template is fitted in intensity and width to match that portion of the $\left[\mathrm{O}_{1}\right]$ profile not contaminated by $\left[\mathrm{S} \mathrm{III}\right.$ emission. (The $\left[\mathrm{O}_{\mathrm{I}}\right]$ and $[\mathrm{O} \mathrm{III}]$ profiles are similar to each other in Mrk 79 and NGC 5548.) To deblend [S III] from [O I] in NGCC 4151, we shifted and scaled the $\left[\mathrm{O}_{\mathrm{I}}\right]+[\mathrm{S} \mathrm{III]}$ blend itself to model the $[\mathrm{S} \mathrm{III}]$ profile and subtracted the result from the $\left[\mathrm{O}_{\mathrm{I}}\right]+[\mathrm{S}$ III] blend. To subtract [O $\mathrm{O}] \lambda 6363$ from [Fe x] $\lambda 6374$, we used the profile of $\left[\mathrm{O}_{\mathrm{I}}\right] \lambda 6300$ scaled by 0.33 , where the scale factor is derived from atomic parameters. To deblend [S II] $\lambda \lambda 6716,6731$, we used an iterative procedure with the blended $\lambda 6731$ profile as an initial template. We fitted the blended $\lambda 6731$ profile to that of the blended $\lambda 6716$ profile with an intensity scale factor as the single free parameter. The resulting profile is a model of a deblended $\lambda 6716$ profile. The model is poorly determined because the template $\lambda 6731$ profile is highly contaminated by $\lambda 6716$ emission. Nevertheless, the model $\lambda 6716$ profile is subtracted from the blend at $\lambda 6716$, resulting in a partially deblended $\lambda 6731$ profile. The procedure is then repeated using the partially deblended $\lambda 6731$ profile as a template. The result is a better model of a deblended $\lambda 6716$ profile than that of the previous iteration because the $\lambda 6731$ template is less contaminated by $\lambda 6716$ emission. More than three iterations only degraded the $\mathrm{S} / \mathrm{N}$ with little or no improvement in the deblending.

We did not apply a formal deblending procedure to reconstruct profiles of [N $\mathrm{II}] \lambda 6548, \mathrm{H} \alpha$ (narrow component), and $[\mathrm{N}$ II $] 26584$. The only objects for which we attempted to analyze these profiles were those in which the narrow components were not blended with each other and the underlying component of broad $\mathrm{H} \alpha$ could be discerned by visual inspection and subtracted off in much the same way we subtracted off the continuum in other lines. The $\mathrm{H} \beta$ profile was used merely as a guide for the "continuum placement."

An atlas of the line profiles extracted from the reduced spectra is given in Figures 1-6. All the profiles presented here are normalized to the peak. Lower resolution studies, in which a large spectral range was obtained on a single night, resulted in more reliable relative flux estimates than did our own study. We include these relative flux estimates in Table 1. The dereddened emission-line fluxes for MCG 8-11-11, Mrk 79, Mrk 704, Mrk 841, and NGC 5548 are from Cohen (1983), and those (not corrected for reddening) 
TABLE 1

Narrow Emission-Line Flux Relative to $\mathrm{H} \beta$

\begin{tabular}{|c|c|c|c|c|c|c|}
\hline Line & MCG 8-11-11 & Mrk 79 & Mrk 704 & Mrk 841 & NGC 4151 & NGC 5548 \\
\hline$[\mathrm{O}$ II $] \lambda 3727 \ldots \ldots \ldots$ & 3.24 & 2.20 & 0.66 & 0.99 & 1.47 & 1.33 \\
\hline$[\mathrm{Fe}$ viI $] \lambda 3760 \ldots \ldots$ & 0.21 & 0.25 & 1.06 & 0.47 & 0.14 & 0.52 \\
\hline [Ne III] $\lambda 3869 \ldots \ldots$ & 1.46 & 1.57 & 1.25 & 1.62 & 1.35 & 1.88 \\
\hline$[\mathrm{O}$ III $] \lambda 4363 \ldots \ldots$. & 0.32 & 0.48 & 0.53 & 0.18 & 0.34 & 1.23 \\
\hline He II $\lambda 4686 \ldots \ldots \ldots$ & 0.16 & 0.25 & 0.43 & 0.32 & 0.15 & 0.26 \\
\hline H I $\lambda 4861 \ldots \ldots \ldots$ & 1.00 & 1.00 & 1.00 & 1.00 & 1.00 & 1.00 \\
\hline$[\mathrm{O}$ iII $] \lambda 5007 \ldots \ldots$ & 8.46 & 10.11 & 6.45 & 11.08 & 15.29 & 9.61 \\
\hline$[\mathrm{Fe}$ vII $] \lambda 5721 \ldots \ldots$ & 0.08 & 0.17 & 0.52 & 0.07 & 0.11 & 0.10 \\
\hline He I $\lambda 5876 \ldots \ldots \ldots$ & 0.10 & 0.13 & 0.11 & 0.17 & 0.15 & 0.07 \\
\hline$[\mathrm{Fe}$ vII] $\lambda 6087 \ldots \ldots$ & 0.10 & 0.28 & 0.74 & 0.30 & 0.22 & 0.35 \\
\hline$\left[\begin{array}{ll}O & 1\end{array}\right] \lambda 6300 \ldots \ldots \ldots$ & 0.40 & 0.36 & 0.16 & 0.32 & 1.06 & 0.26 \\
\hline$[\mathrm{S}$ III] $\lambda 6312 \ldots \ldots \ldots$ & $\ldots$ & 0.05 & 0.12 & 0.04 & 0.03 & 0.03 \\
\hline$[\mathrm{Fe} \times] \lambda 6374 \ldots \ldots$ & 0.06 & 0.11 & 0.49 & 0.11 & 0.12 & 0.08 \\
\hline $\mathrm{H}$ I $\lambda 6563 \ldots \ldots \ldots$ & 2.08 & 2.93 & 2.62 & 2.87 & 5.29 & 2.43 \\
\hline$[\mathrm{N}$ II] $\lambda 6584 \ldots \ldots \ldots$ & 2.06 & 2.16 & 0.91 & 1.08 & 2.24 & 1.10 \\
\hline 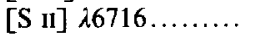 & 0.58 & 0.72 & 0.22 & 0.35 & 0.82 & 0.21 \\
\hline$[\mathrm{S}$ II $] \lambda 6731 \ldots \ldots \ldots$ & $\ldots$ & 0.72 & 0.31 & 0.37 & 1.00 & 0.20 \\
\hline
\end{tabular}

for NGC 4151 are from Boksenberg et al. (1975). The [Fe x] $\lambda 6374$ line strength in NGC 4151 was determined from the $\lambda 6374$ to 26300 line strength ratio of Veilleux (1988) and the $\lambda 6300$ line strength of Boksenberg et al. (1975), as $\lambda 6374$ was not included in the latter study. These line strengths have implications for some of the models discussed in Paper II. For possible future reference, we have also included [S III] $\lambda 6312$ relative line strengths based on our measured [S III] $\lambda 6312$ to $[\mathrm{O} \mathrm{I}] \lambda 6300$ line strength ratios and the previously published $[\mathrm{OI}]$ line strengths.

\section{GENERAL IMPLICATIONS FOR THE NLR}

Before discussing interpretations of the line profiles, we summarize some important characteristics of NLR model profiles that should not be overlooked. The basic goal is to provide interpretations that stem directly from the observed profiles to the extent possible, with only minimal reference to specific models. The most important premise is that the preponderance of blueward asymmetry constrains the highest velocity clouds to move mostly in the radial direction and precludes the presence of a broad distribution of velocities at each radial coordinate, $r$ (asymmetric distributions of gas would produce red and blue asymmetries with equal likelihood). Neglecting obscuration, a spherical shell of delta function-emitting clouds moving radially with velocity $v_{0}$ yields a boxcar profile with half-width $v_{0}$. As can be inferred directly by visual inspection, replicating the observed profiles requires that either boxcar profiles with a range of velocity width must be superposed, or else assumptions such as purely radial cloud flow or a spherical NLR must be relaxed.

In MC we showed that the profile wings are not sensitive to either the orientation or the opening angle of a biconical line-emitting structure (with the caveat that the velocity field is renormalized appropriately when the line of sight falls outside the biconical structure), even though the profile core is. In Moore $(1994,1995)$, it is demonstrated that the blue wing is not very sensitive to details of the obscuration, whether internal or external to the emitting clouds. Thus, it is possible to interpret the blue wings readily in the context of a simplified model NLR even though collimated emission and obscuration are likely present. This point is worth emphasizing because it follows then from the absence of a broad distribution of cloud velocities at each $r$ (see above) that the range in height and in velocity width of boxcar profiles that must be superposed to replicate the observed blue wings relates directly to the spatial dependence of the line emission. For lines that are useful diagnostics of electron density $\left(n_{e}\right)$, column density $\left(N_{\text {coo }}\right)$, and/or ionization parameter $(\Gamma)$, the spatial dependence of the line emission in turn corresponds directly to how $n_{e}, N_{\text {col }}$, and $\Gamma$ vary spatially throughout the NLR. The effects of spatial variations in $n_{e}, N_{\text {col }}$, and $\Gamma$ are discussed qualitatively when we discuss the profiles of individual objects. The effects are illustrated more explicitly (though less independent of detailed modeling) in Paper II.

It is an observational fact that, in some objects, line profiles of high and low IP have very similar blue wings (see the discussion in MC as well as the profiles presented in $\$ 3$ and in Veilleux 1991a). It follows that if the velocity field is at all close to monotonic, then there is emission into lines of both high and low IP at each $r$, which could arise in either of two scenarios.

The first possible scenario is that individual clouds are stratified in ionization. The second possible scenario is that there is a broad distribution in the electron density of unstratified clouds and, therefore, in $\Gamma$ at each $r$. Throughout this paper, we use the term "stratified cloud" to denote a single cloud of sufficient ionization stratification to emit $[\mathrm{Fe} \mathrm{X}]$ and/or $[\mathrm{Fe} \mathrm{VII}]$ lines from the front as well as $[\mathrm{O} \mathrm{I}]$ and [S II] lines from the back. An "unstratified cloud" is optically thin to the photoionizing continuum so that a single ionization state describes the entire cloud. It is worth pointing out that blueward asymmetry and stratified clouds are consistent with either inflow or outflow according to whether the obscuration is mostly internal or external, respectively. Because the applicability of the first scenario is fundamental to our detailed modeling (Paper II) and to several of our conclusions, we outline here why we believe the first scenario applies. We note, however, that work favoring the second scenario is underway (Komossa \& Schulz 1994; Tsvetanov et al. 1994). Our arguments for stratified clouds are as follows:

1. Clouds of very different $n_{e}$, but at the same $r$, cannot all be pressure confined. Because the equilibrium temperature of photoionized gas varies only slightly with $n_{e}$ (MC), there is a narrow range of $n_{e}$ at each $r$ that can be in pressure equilibrium either with the intercloud medium 
(ICM) or with a magnetic field. Because the cloud expansion timescale is a small fraction of the crossing time, a broad distribution of $n_{e}$ at each $r$ would require that clouds are continually created and destroyed (this has been suggested for broad-line region [BLR] clouds based on a stability analysis [Krinsky \& Puetter 1992]).

2. A contrived distribution of $n_{e}$ must be invoked for the second scenario so that each $n_{e}$ contributes comparable flux to the appropriate emission line. Considering that the volume emissivity for optically thin gas is $j \propto n_{e}^{2}$, and the emission per fixed mass is proportional to $n_{e}$, arranging for clouds differing by several decades in $n_{e}$ to produce comparable emission-line flux at each $r$, as observations require, is not trivial.

3. An inflow model requires stratified clouds to explain the preponderance of blueward asymmetry. An outflow model (with external obscuration) can accommodate unstratified clouds, but a mechanism must be found to outwardly accelerate clouds of very different density to nearly the same velocity. Otherwise, a broad distribution of projected velocity at each $r$, and consequently symmetric profiles, would result. In principal, clouds of very different $n_{e}$ can be accelerated to similar velocities over very different length scales. This scenario could produce distinct coexisting velocity fields, with different lines coming from different underlying cloud populations at different $r$. However, to account for the striking similarity in the profiles of different lines in some objects, this exceedingly contrived scenario requires that the variation of emission-line flux with projected velocity is virtually identical for lines in which the variation of flux with $r$ is very different.

4. While lines of high and low IP/ $n_{\mathrm{cr}}$ are very similar in some objects, the high IP $/ n_{\mathrm{cr}}$ lines are systematically broader in other objects. Both types of objects would not be explained naturally by a broad distribution in $n_{e}$ and $\Gamma$ at each $r$. Actually, both types of objects are also not easily explained in the classical picture of the NLR in which the highest velocity clouds are at small $r$, where lines of low $n_{\mathrm{cr}}$ are collisionally suppressed, resulting in systematically narrower low $n_{\text {cr }}$ lines. To explain both types of objects in the classical picture of the NLR, the two types of objects must differ in the maximum $n_{e}$ by several decades, which is the relevant range of $n_{\mathrm{cr}}$. In Paper II, we present models in which line width correlations are a natural consequence of outward acceleration and of $N_{\text {col }} \propto r^{-4 / 3}$, the latter resulting from spherical clouds of constant mass and with constant ionization parameter. In these models, objects in which the low-ionization potential lines are broad and narrow differ in $N_{\text {col }}$ by factors of only order unity.

\section{INDIVIDUAL OBJECTS}

\section{1. $M C G 8-11-11$}

The profiles extracted from MCG 8-11-11 spectra are plotted in Figure 1. We used the [O I] $\lambda 6300$ line rather than [O III] $\lambda 5007$ to estimate the systemic velocity of this object because $\lambda 6300$ is more symmetric than $\lambda 5007$, and an asymmetric profile yields an underestimated systemic velocity due to obscuration effects. We used the centroid rather

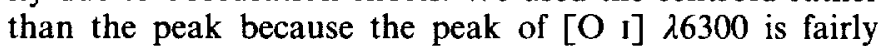
flat-topped, so the peak location is ambiguous.

The [O II] $\lambda 3727$ profile is a composite of the doublet, $\lambda 3726+\lambda 3729$, corresponding to a separation of $217 \mathrm{~km}$ $\mathrm{s}^{-1}$. The blue wing of $\lambda 3727$ arises mostly from $\lambda 3726$ emis- sion. Clearly, from the apparent extent of the blue wing in Figure $1 a$, there are clouds with $v \approx-1000 \mathrm{~km} \mathrm{~s}^{-1}$ appreciably emitting $\lambda 3726$. The critical density of this line is $3 \times 10^{3} \mathrm{~cm}^{-3}$, so the electron density of the $v \approx-1000 \mathrm{~km}$ $\mathrm{s}^{-1}$ clouds emitting $\lambda 3726$ does not greatly exceed $10^{4}$ $\mathrm{cm}^{-3}$

As is the case with the profiles of Veilleux (1991a), the presence of a substantial velocity gradient across the NLR can be inferred by visual inspection of the profiles. In particular, note that a similar range of radial velocity can be inferred for all the profiles shown in Figure 1 (i.e., for each of the profiles, a substantial range in velocity width of boxcar profiles must be superposed to replicate the blue wing). In the absence of a broad distribution of cloud velocity at each $r(\S 3)$, the similar range of radial velocity corresponds to similar spatial extents for the line emission. In the case of $[\mathrm{Fe} \mathrm{VII}] \lambda 6087$, the similar spatial extent suggests a roughly constant ionization parameter throughout the NLR because [Fe VII] $\lambda 6087$ is much more sensitive to ionization parameter than the other lines (MC). In the case of [O I] $\lambda 6300$, the similar spatial extent suggests that the clouds are also optically thick to the photoionizing continuum throughout the NLR. The same consideration could also suggest that the low-density limit $\left(n_{e} \ll n_{\mathrm{cr}}\right)$ is valid throughout the NLR for even the lowest critical density lines ([O II] $\lambda 3727$ and $[\mathrm{S} \mathrm{II}] \lambda \lambda 6716,6731)$ because the effects of collisional de-excitation are apparent in neither the wings nor in the cores of these lines. Although the effects of collisional de-excitation on the profiles are illustrated explicitly in the detailed kinematical models of Paper II, they can also be discerned qualitatively as follows. If the NLR is spherical, and if obscuration can be neglected, and if the velocity field is radial even for the lowest velocity clouds, then the line profile is a simple superposition of boxcar profiles. Collisional de-excitation effects in the highest velocity clouds would be evident if the low critical density line profiles were lacking a contribution from the highest velocity boxcar profiles (i.e., the profile wings). Collisional de-excitation effects in the lowest velocity clouds would be evidenced by a broad flat profile peak (there would be no contribution from the lowest velocity boxcar profiles). Absence of collisional deexcitation effects throughout the [O II] $\lambda 3727$ and [S II] $\lambda \lambda 6716,6731$ profiles would then limit the cloud electron density to $n_{e} \lesssim 10^{4} \mathrm{~cm}^{-3}$ throughout the NLR. However, the NLR model we favor includes $n_{e} \approx 10^{6} \mathrm{~cm}^{-3}$ gas (see Paper II, MC, and Moore 1994) and requires emission from low-density and low-velocity gas outside the (parameter space of the) NLR model to account for the [O $\mathrm{II}] \lambda 3727$ and $[\mathrm{S}$ II] $\lambda \lambda 6716,6731$ profile cores.

\section{2. $M r k 79$}

The profiles extracted from Mrk 79 spectra are plotted in Figure 2. We have presented the profile of [S II] $\lambda 6731$ rather than that of [S II] $\lambda 6716$ because the latter is badly corrupted by the $B$-band absorption feature. The [Fe X] $\lambda 6374$ profile is of such poor quality that almost nothing can be said about the NLR based on this profile except that there are [Fe $\mathrm{x}]$ ג6374-emitting clouds with $v \approx-1000 \mathrm{~km}$ $\mathrm{s}^{-1}$ and that there is some range of (radial) velocity sampled by the $\lambda 6374$-emitting clouds because the profile is not a single boxcar profile. However, $\lambda 6374$ is so sensitive to ionization parameter (MC) that even this small piece of information is useful and implies that the ionization parameter is roughly constant over whatever range of velocity can be 

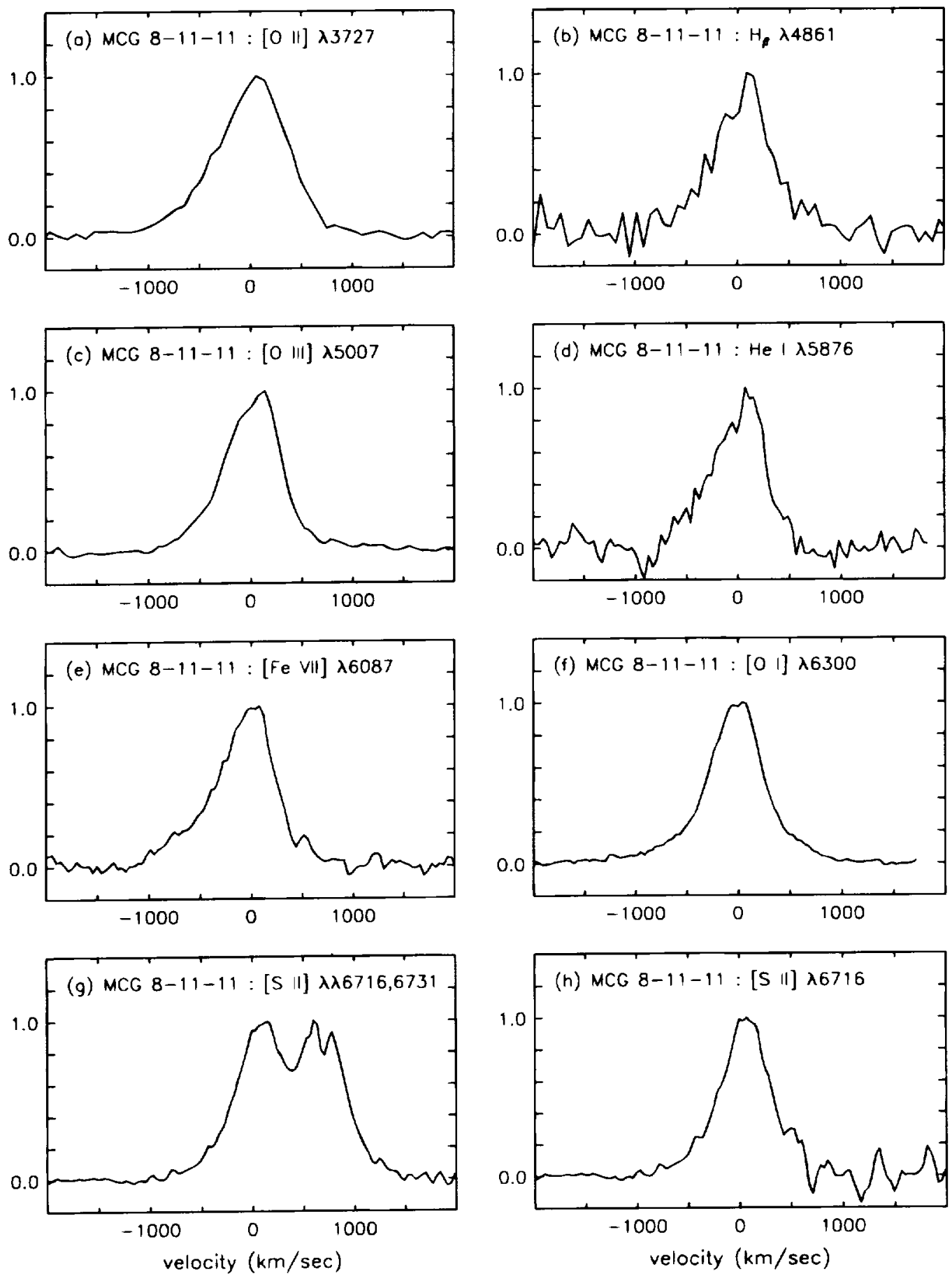

FIG. 1.-Narrow emission-line profiles extracted from spectra of MCG 8-11-11. A scenario in which the NLR can be decomposed into physically distinct regions in which the low-ionization potential/critical density lines and the high-ionization potential/critical density lines are formed is inconsistent with the similarity in the profiles shown here. To account for the similarity in the profiles requires significant emission in each line over a large fraction of the NLR. In particular, note the similar velocity ranges sampled by $\left[\mathrm{S} \mathrm{II}_{\mathrm{I}} \lambda 6716\right.$ and $\left[\mathrm{O}_{1}\right] \lambda 6300$. These lines differ by three decades in critical density. Panels $(g)$ and $(h)$ represent [S II] before and after deblending, respectively. The trough of the $B$-band absorption feature coincides with the peak of $\lambda 6731$. Due to the coincidence in wavelength, the $B$-band correction is poor, as evidenced by the residual feature at the peak of $\lambda 6731$. Although $\lambda 6731$ was excluded from further analysis, profile parameters were calculated, nonetheless, and they were in reasonable agreement with those of $\lambda 6716$.

inferred from the profile. Note that the [Fe vII] $\lambda 6087$ profile has an extended blue wing that is not present in other profiles (we exclude the $[\mathrm{Fe} \mathrm{x}] \lambda .6374$ profile from the discussion owing to the very low $\mathrm{S} / \mathrm{N}$ in the wings). The FWHM of [Fe vII] $\lambda 6087$ is similar to that of [O III $\lambda 5007$, but a width parameter defined at a lower fraction of the peak intensity is significantly larger for $[\mathrm{Fe} \mathrm{vII]}$ than for
[O $\mathrm{O}$ III]. Our favored models $\left(n_{e} \propto r^{-2}, v \propto r^{1 / 2}\right.$, and $N_{\text {col }} \propto r^{-4 / 3}$ ) show the same effect as well (Paper II).

In both objects, MCG 8-11-11 and Mrk 79, the [Fe VII] $\lambda 6087$ profile is broad. The [O $\mathrm{O}] \lambda 6300$ profile is either broad (MCG 8-11-11) or narrow (Mrk 79). When [O I] is broad, $[\mathrm{S} \mathrm{II}]$ is also broad, and when $[\mathrm{O} \mathrm{I}]$ is narrow, [S II] is also narrow. This is consistent with the trend discussed 

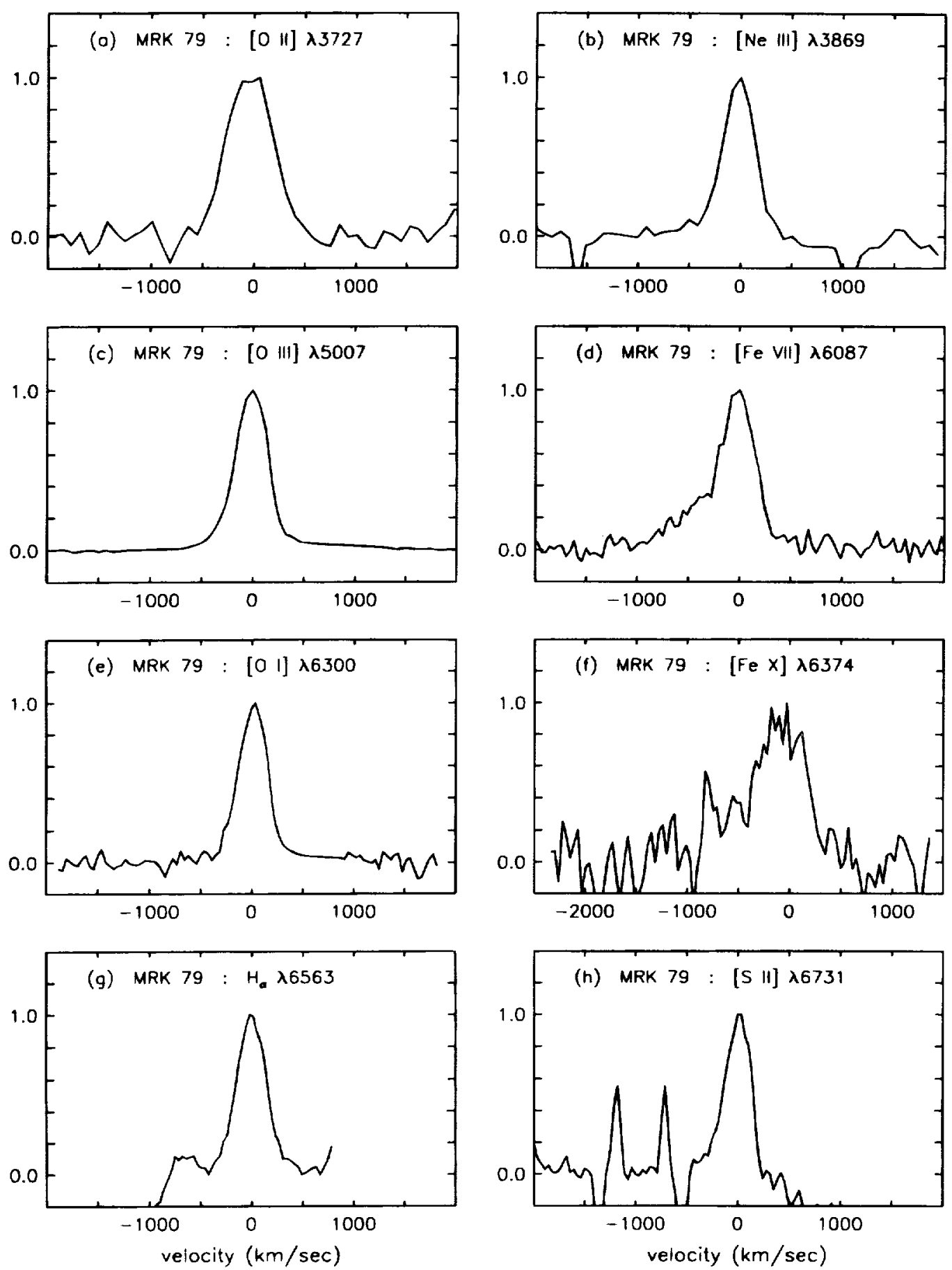

Fig. 2.-Narrow emission-line profiles extracted from spectra of Mrk 79. Note that in Mrk 79, unlike in MCG 8-11-11, [O I] and [S II] are both narow relative to [Fe VII]. We explain the difference between MCG 8-11-11 and Mrk 79 in terms of identical NLRs except that clouds at the inner radius of MCG 8-11-11 start out with somewhat higher column density than those of Mrk 79. In this scenario for Mrk $79, N_{\text {col }} / N_{\text {min }}$ decreases to less than one before the clouds reach their maximum velocity at the outer radius, while in the MCG 8-11-11 scenario, $N_{\text {sol }} / N_{\min }>1$ throughout the NLR.

in $\mathrm{MC}$ for a much larger sample $(\approx 50)$ of objects. The trend for $\left[\mathrm{O}_{\mathrm{I}}\right]$ and $[\mathrm{S} \mathrm{II}]$ to have similar widths poses a difficulty for interpreting line width correlations as fundamentally with $n_{\mathrm{cr}}$ because these lines differ in $n_{\mathrm{cr}}$ by three decades.

We can explain the difference between MCG 8-11-11 and Mrk 79 in terms of identical NLRs, except that clouds at the innermost radius in MCG 8-11-11 start out with somewhat higher column density than those in Mrk 79. In Mrk 79, $N_{\text {col }}$ decreases to less than $N_{\text {min }}$ before the clouds reach their maximum velocity at the outermost radius, while in MCG $8-11-11, N_{\text {col }} / N_{\min }>1$ throughout the NLR. We define $N_{\min }$ as the minimum column density needed for an [O I] 26300 -emitting zone to exist in the back of the clouds. Referring to the models of Paper II, MCG 8-11-11 corresponds to model 3 and Mrk 79 corresponds to model 4 . We postulate that our model for Mrk 79 is representative of objects that show correlations of line width with ionization potential, while our model for MCG 8-11-11 is representative of objects that do not. 


\section{3. $M r k 704$}

The profiles extracted from Mrk 704 spectra are plotted in Figure 3. The systemic velocity was estimated from the peak of $[\mathrm{O}$ III $] \lambda 5007$. Note the extended blue wing in the [Fe vII] profile, from which it is clear that the [Fe VII]emitting clouds sample a wide range of velocity. Of even greater significance, a substantial range of velocity can also be inferred from the $[\mathrm{Fe} \mathrm{x}] \lambda 6374$ profile, which is extremely sensitive to ionization parameter (MC). Because of the wide range of cloud velocity that can be inferred directly from the observed $[\mathrm{Fe} \mathrm{x}] \lambda 6374$ profile, our case for constant ioniza- tion parameter is strongest for Mrk 704, NGC 4151, and NGC 5548.

As is the case for Mrk 79, the extended blue wing in the [Fe VII] profile of Mrk 704 is not evident in the profiles of $\left[\mathrm{O}_{\mathrm{I}}\right] \lambda 6300$ and $[\mathrm{S}$ II] $\lambda \lambda 6716,6731$. The obviously low quality of the $\left[\mathrm{O}_{\mathrm{I}}\right]$ and $[\mathrm{S} \mathrm{II}]$ profiles precludes a more definitive statement, however. The $\left[\mathrm{O}_{\mathrm{I}}\right]$ and $[\mathrm{S} \mathrm{II}]$ profiles of Mrk 79 are suggestive of the following interesting effect, which is evident in model 4 of Paper II though the effect is inconclusive here owing to the low quality of these profiles. The effect is that a width parameter defined in the wings is
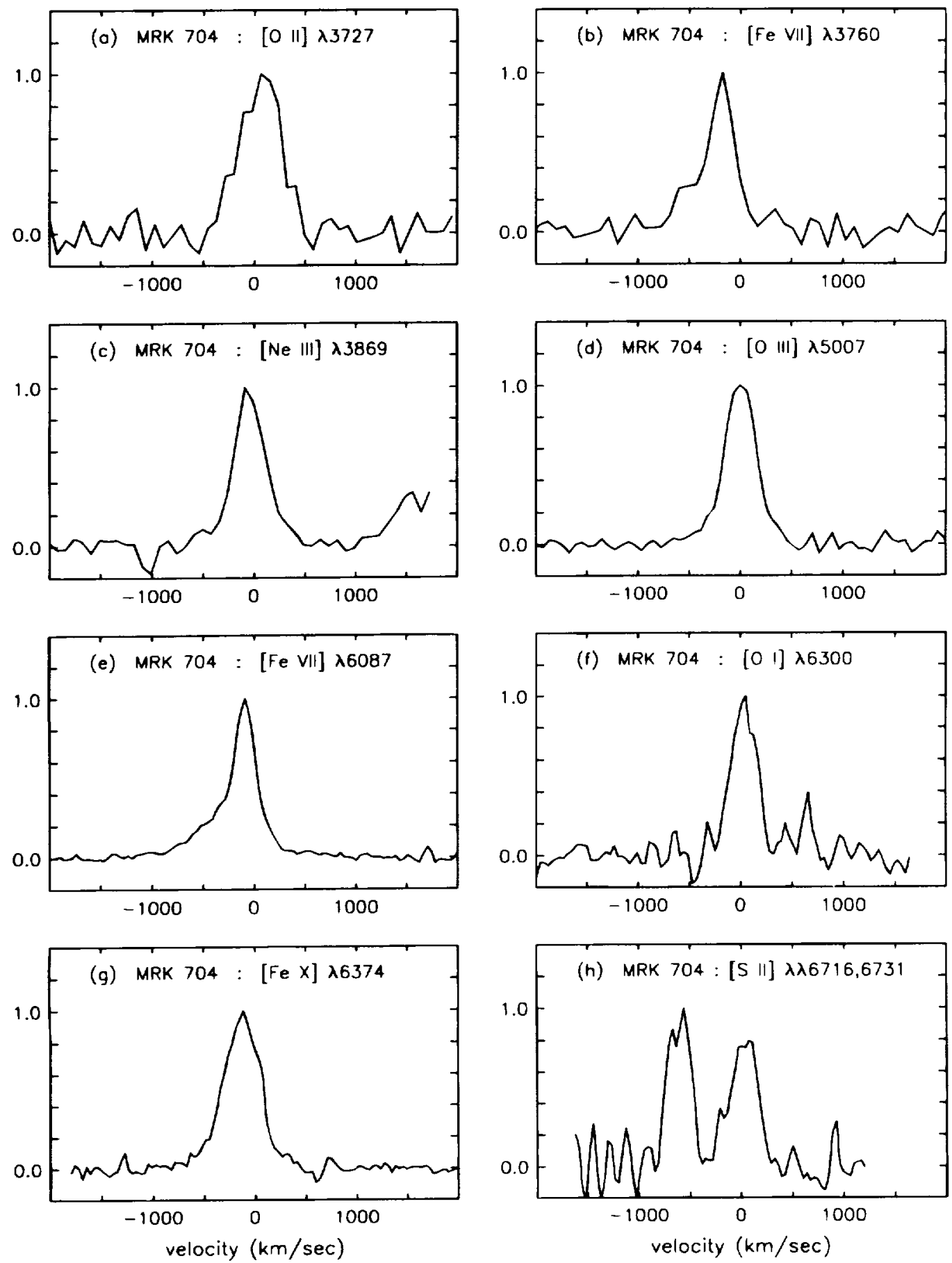

Fig. 3.-Narrow emission-line profiles extracted from spectra of Mrk 704. As is the case in Mrk 79, the extended blue wing of the [Fe viI] profile of Mrk 704 is not evident in the [ $\left.\mathrm{O}_{1}\right]$ and $\left[\mathrm{S} \mathrm{II}_{1}\right]$ profiles. 
larger for [ $\mathrm{Fe} \mathrm{VII}]$ than for [O $\mathrm{O}]$ and [S II], even though the FWHM is about the same, and the width at any fraction of the peak intensity is similar for $[\mathrm{O} \mathrm{I}] \lambda 6300$ and $[\mathrm{S} \mathrm{II}] \lambda 6716$.

\section{4. $M r k 841$}

The profiles extracted from Mrk 841 spectra are plotted in Figure 4. The systemic velocity was estimated from the peak of $\left[\mathrm{O}_{\mathrm{I}}\right] \lambda 6300$. Note the very extended $(\gtrsim 1000 \mathrm{~km}$ $\mathrm{s}^{-1}$ ) blue wing of the [Fe vII] $\lambda 6087$ profile. This wing is not present in other profiles (the $[\mathrm{Fe} \mathrm{x}]$ profile is inconclusive).
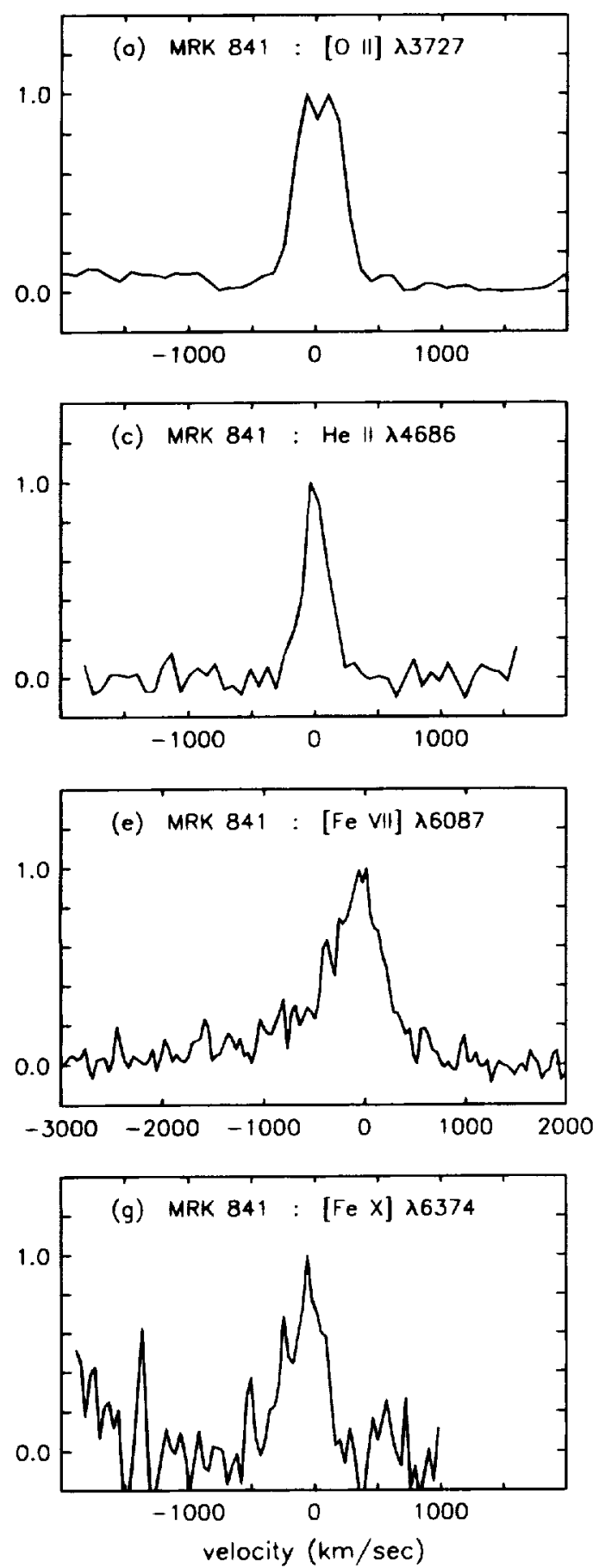

For Mrk 841, unlike Mrk 79 and Mrk 704, the width at any fraction of the peak intensity is greater, almost up to the peak itself, for $[\mathrm{Fe} \mathrm{VII}]$ than for $\left[\mathrm{O}_{\mathrm{I}}\right]$ and $[\mathrm{S} \mathrm{II}]$. Note also that the [S II] profiles are narrow, and the $[\mathrm{O} \mathrm{I}]$ profile is narrow as well, in keeping with the trend discussed here and in MC. Thus, we assert that Mrk 841 is best explained by model 4.

\section{5. $N G C 4151$}

The profiles extracted from NGC 4151 spectra are plotted in Figure 5. We include two profiles (Figs. $5 a-5 b$ ) of
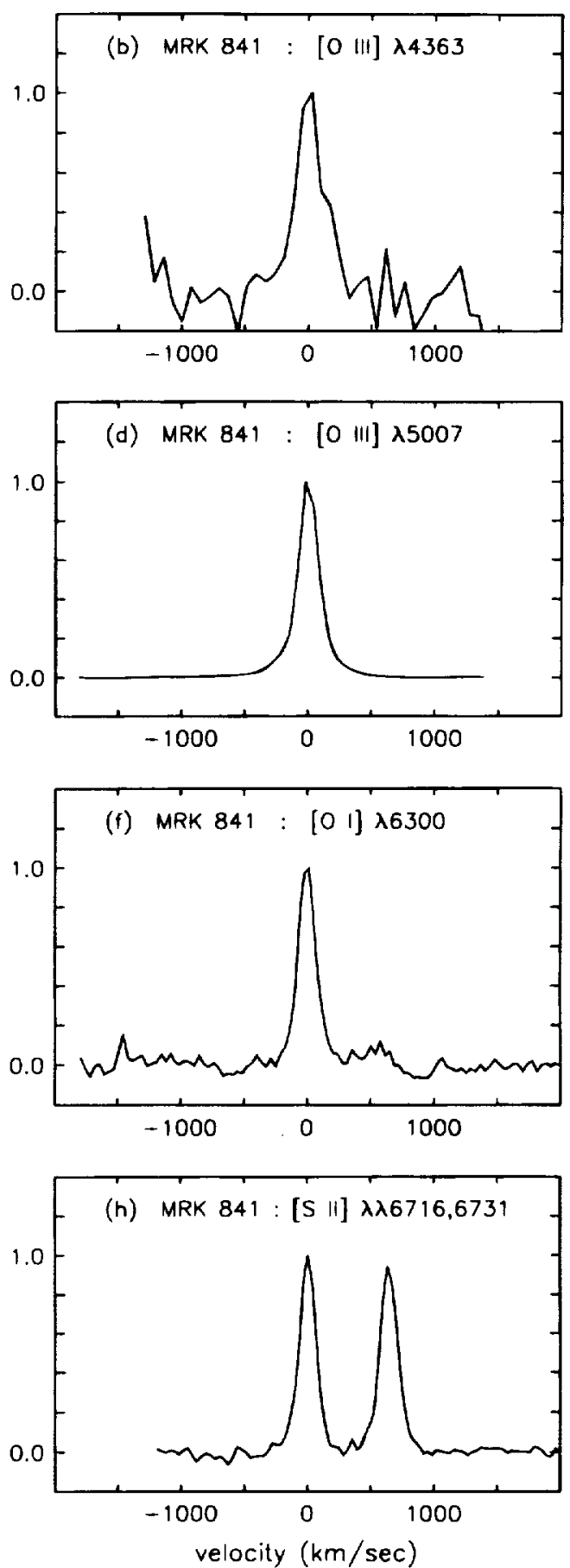

FIG. 4.-Narrow emission-line profiles extracted from spectra of Mrk 841. Note that [O I] and [S II] are both very narrow relative to [Fe viI]. This is in keeping with a general trend: when $[\mathrm{O} \mathrm{I}]$ is narrow, $[\mathrm{S} \mathrm{II}]$ is narrow as well, and when $[\mathrm{O} \mathrm{I}]$ is broad, [ $\mathrm{S} \mathrm{II}]$ is broad also. These lines differ in critical density by three decades, however. 

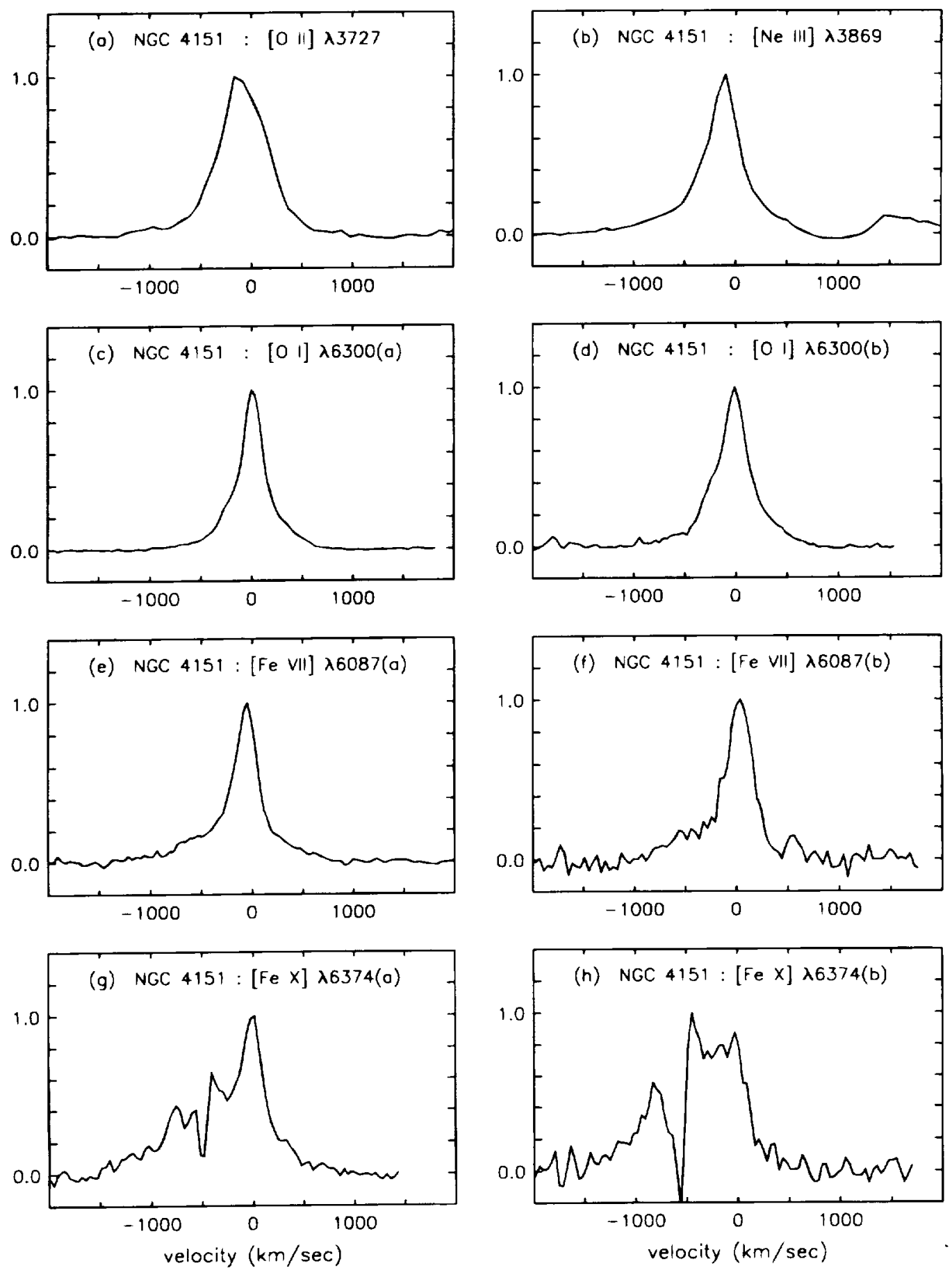

FIG. 5.-Narrow emission-line profiles extracted from spectra of NCG 4151. We include two profiles, $(a)$ and $(b)$, of [O I], [Fe vil], and [Fe $\mathrm{x}$ ] corresponding to two observations 105 days apart because the Fe lines of NGC 4151 have been reported in the literature as possibly variable (Penston et al. 1984). Note that the [Fe VII] profiles of NGC 4151 and Mrk 704 are virtually identical, suggesting a very simple common acceleration mechanism. Note also that the $[\mathrm{Fe} \mathrm{x}]$ profile has the same overall characteristics as the [Fe vII] profile of any object presented thus far. This would not likely result if $[\mathrm{Fe} \mathrm{x}]$ and $[\mathrm{Fe}$ VII $]$ were emitted in physically distinct regions.

[O I ] $\lambda 6300,[\mathrm{Fe} \mathrm{vII}] \lambda 6087$, and [Fe x] $\lambda 6374$ corresponding to two observations 105 days apart. NGC 4151 is highly variable in its continuum luminosity, and the forbidden $\mathrm{Fe}$ lines of NGC 4151 have been reported as possibly variable (Penston et al. 1984). We see no compelling evidence of variability in the profiles here (of course, our time baseline is much smaller than the 3 year baseline of Penston et al.).

As in every object presented thus far, the $[\mathrm{Fe} \mathrm{VII}]$ profile of NGC 4151 shows a very extended ( $\left.\gtrsim 1000 \mathrm{~km} \mathrm{~s}^{-1}\right)$ blue wing. The similarity in the $[\mathrm{Fe}$ VII $]$ profiles of NGC 4151
(Fig. 5a) and of Mrk 704 is striking. The profiles are virtually identical. In all five objects whose profiles we have presented thus far, the [ $\mathrm{Fe}$ vII] profiles are very similar and do not differ even in an overall scale factor in the velocity width. In every object, we can infer [Fe VII] emission out to $v \approx 1000 \mathrm{~km} \mathrm{~s}^{-1}$ and not much higher. The remarkable similarity in the [Fe VII] profiles of each object suggests a very simple common acceleration mechanism. Likewise, Whittle (1991) has pointed out previously that the similarity in the [O III $\lambda 5007$ profiles from a large number of objects 

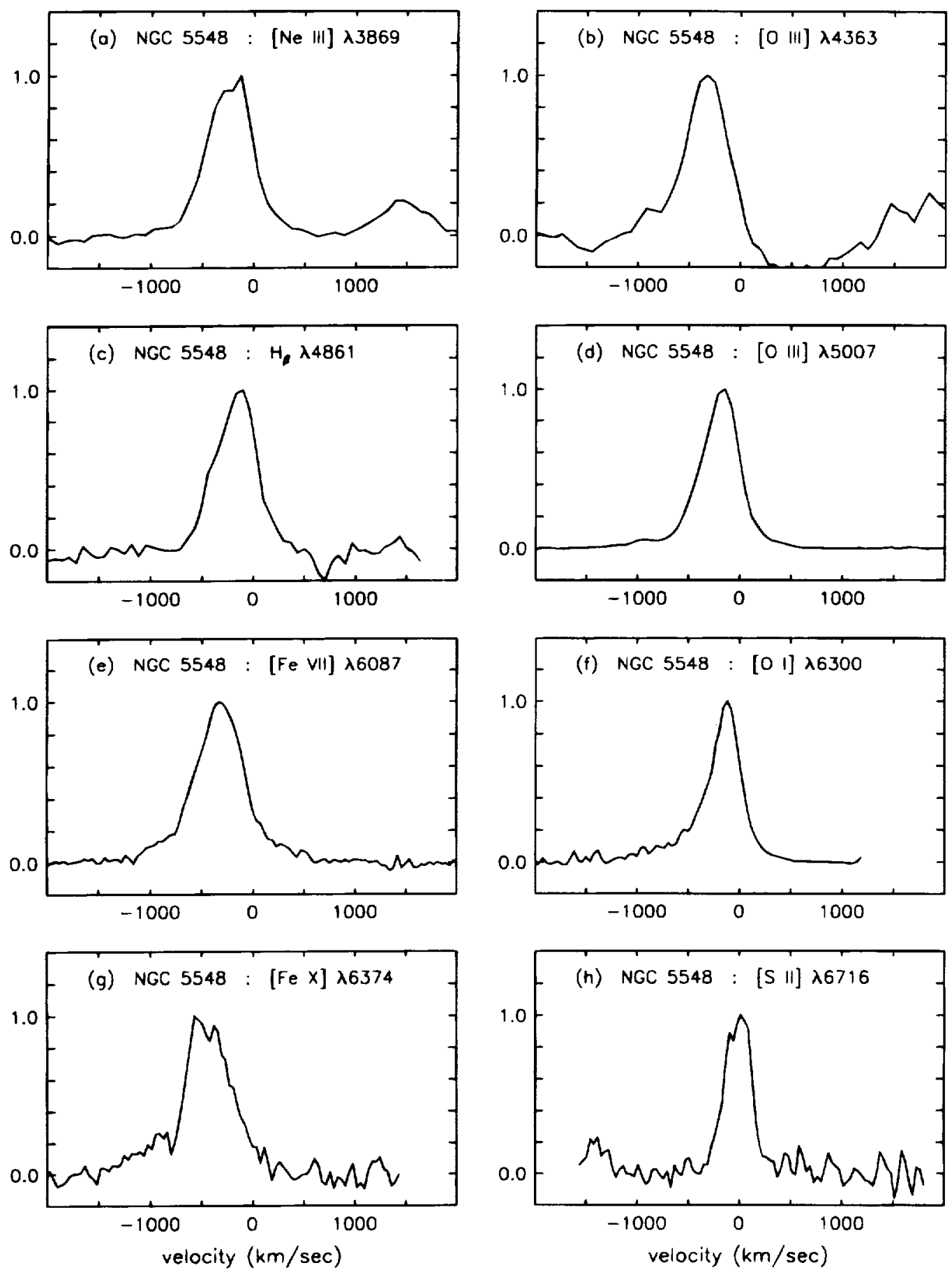

Fig. 6.-Narrow emission-line profiles extracted from spectra of NGC 5548. Note that the [ $\left.\mathrm{O}_{1}\right]$ profile is broad, but the [S $\left.\mathrm{II}\right]$ profile is narrow. This is in contrast to a general trend discussed here and in MC.

suggests a common acceleration mechanism for the $\lambda 5007$ emitting gas.

The similarity in the [Fe vII] $\lambda 6087$ profiles of NGC 4151 and Mrk 704 has possible implications concerning how representative our sample of Seyfert 1 galaxies is. Because the sample was chosen with the goal of obtaining high-quality [Fe vII] $\lambda 6087$ and, when possible, [Fe x] $\lambda 6374$ profiles, selection was biased toward objects with strong $\lambda 6087$ and $\lambda 6374$ emission. It is quite possible that the same properties of the same that allow us to obtain reliable $\lambda 6087$ and $\lambda 6374$ profiles also render the sample unrepresentative of Seyfert 1 galaxies in general. The forbidden Fe line strengths of NGC
4151 are more representative of a typical Seyfert 1 galaxy than those of Mrk 704. We were able to extract reliable $\lambda 6087$ and $\lambda 6374$ profiles of NGC 4151 because our spectra have high $\mathrm{S} / \mathrm{N}$ due to this object's brightness. Of the six objects in our sample, the $\lambda 6087$ line of Mrk 704 is the strongest relative to $\mathrm{H} \beta(0.87)$, while that of NGC 4151 is the weakest $(0.07)$. The object in our sample with the strongest 26087 line and an object that is representative in its $\lambda 6087$ line strength of a typical Seyfert 1 galaxy have virtually identical profiles, which suggests that the density and velocity profiles throughout the NLR are very similar in both objects. That is, we do not infer any important differ- 
ences between Mrk 704 and NGC 4151 other than the different $[\mathrm{Fe} \mathrm{VII}]$ line strengths. The argument could be tested by selecting at random one or two objects with weak [Fe VII] line strengths and investing the telescope time required to obtain $\mathrm{S} / \mathrm{N}$ comparable to that of our profiles of [Fe VII] $\lambda 6087$ in both Mrk 704 and NGC 4151. We note also that, although Veilleux (1991a) did not include any $[\mathrm{Fe} \mathrm{x}] \lambda 6374$ profiles and included [Fe vII $] \lambda 6087$ profiles for only Mrk 359 and NGC 4151, the lower ionization line profiles in his sample are similar to those in our own sample.

Any of the [Fe VII] profiles presented thus far will suffice more or less as an archetypical [Fe vII] $\lambda 6087$ profile. The [Fe x] $\lambda 6374$ profile of NGC 4151 has the same overall characteristics as the [Fe VII $\lambda 6087$ profile of any object. This suggests that $[\mathrm{Fe} \mathrm{x}]$ is emitted in a region that is similar spatially to the region of $[\mathrm{Fe}$ VII] emission rather than from a physically distinct region close to the central source with $n_{e} \approx n_{\mathrm{cr}}=5 \times 10^{9} \mathrm{~cm}^{-3}$. Our case for constant-ionization parameter, based on the forbidden $\mathrm{Fe}$ lines, is probably strongest for NGC 4151. In Paper II we present some models in which ionization parameter decreases outward but not so much that the model [Fe VII] $\lambda 6087$ profile is inconsistent with observations. The $[\mathrm{Fe} \mathrm{x}]$ $\lambda 6374$ profile, on the other hand, is extremely sensitive to ionization parameter. Almost any spatial variation at all in the ionization parameter results in a synthetic $[\mathrm{Fe} \mathrm{x}] \lambda 6374$ profile that is, contrary to observations, different from that of $[\mathrm{Fe} \mathrm{VII}] \lambda 6087$ and other profiles.

NGC 4151 is like MCG $8-11-11$ in that the [Fe VII] and $\left[\mathrm{O}_{1}\right]$ profiles are very similar. Thus, we infer similar spatial extents to the $\left[\mathrm{O}_{\mathrm{I}}\right]$ and [Fe VII] line emission, which requires that either $\left[\mathrm{O}_{\mathrm{I}}\right]$ and $[\mathrm{Fe}$ vII] are emitted from clouds at the same $r$ but with very different $n_{e}$, or else the emitting clouds are individually stratified in ionization. We argued for the latter in $\S 3$. We do not have a spectrum of NGC 4151 that includes the [S II] lines, but the blue wing of the [S II] $\lambda 6716$ profile presented by Veilleux (1991a) is very similar to that of $\left[\mathrm{O}_{\mathrm{I}}\right] \lambda 6300$ (either ours or Veilleux's). Again, this agrees with the trend discussed here and in $\mathrm{MC}$, namely, when $\left[\mathrm{O}_{\mathrm{I}}\right]$ is broad, $[\mathrm{S} \mathrm{II}]$ is broad as well.

\section{6. $N G C 5548$}

The profiles extracted from NGC 5548 spectra are plotted in Figure 6 . The overall characteristics of the [Fe VII] profile from NGC 5548 are consistent with the [Fe vII $]$ profile from the above five objects, although we cannot infer as wide a range of cloud velocity from this profile. The latter could be due to a smaller $v_{\max } / v_{\min }$ for the [Fe VII $] \lambda 6087-$ emitting gas in this object or perhaps to a larger chaotic component of the velocity field. We define $v_{\max }$ as the maximum radial velocity of NLR clouds. It is the velocity at the innermost or outermost NLR radius, according to whether the cloud velocity increases inward or outward. Similarly, $v_{\min }$ is the minimum radial velocity of NLR clouds. When the ratio of the maximum radial velocity in the NLR to the minimum radial velocity $\left(v_{\max } / v_{\min }\right)$ is decreased, the wings are less pronounced, and the result is a profile closer to that of a boxcar function.

Note that our discussion of the $[\mathrm{Fe} \mathrm{x}]$ profile of NGC 4151 applies to NGC 5548 as well. The blue wing of the [Fe X] profile extends out to about $1500 \mathrm{~km} \mathrm{~s}^{-1}$ relative to the systemic velocity (but about $1000 \mathrm{~km} \mathrm{~s}^{-1}$ relative to the peak). Even though $v_{\max } / v_{\min }$ may not be as large for this profile as other profiles presented thus far, a wide range of radial velocity is clearly present in this profile. Thus, the ionization parameter will be fairly constant over much of the NLR in NGC 5548. The blue wing of $\left[\mathrm{O}_{\mathrm{I}}\right]$ is about as extended as that of $[\mathrm{Fe} \mathrm{x}]$ (i.e., significant flux out to a projected velocity of about $-1000 \mathrm{~km} \mathrm{~s}^{-1}$ ), from which we infer that the clouds are also optically thick throughout the NLR.

Note that the $\left[\mathrm{O}_{\mathrm{I}}\right]$ profile is broad, but the $[\mathrm{S}$ II] profile is narrow, in contrast to the general trend discussed here and in MC. As discussed further in $\S 5$, for NGC 5548, the widths of [O I] $\lambda 6300$ and [O III $\lambda 24363$, as well as the median velocity of both these lines, seem to correlate better with critical density than with ionization potential. Because NGC 5548 is unusual in other respects as well, we discuss this object in particular detail in $\S 6$.

\section{PROFILE PARAMETER CORRELATIONS}

So far, the features of the profiles we have emphasized are usually obvious, and we have had little need for numerical values of profile parameters. For example, when we have noted that $\left[\begin{array}{ll}\mathrm{O}_{1}\end{array}\right]$ and $\left[\mathrm{S}_{\mathrm{II}}\right]$ profiles are narrow compared with $[\mathrm{Fe}$ vII $]$, the difference in width is large enough to discern immediately by visual inspection of the profiles. Indeed, much of the discussion of line width correlations that follows is driven by the similarity of $\left[\mathrm{O}_{\mathrm{I}}\right]$ and $[\mathrm{S} \mathrm{II}]$ line widths as discussed in MC. However, profile parameters are useful to confirm continuity in the sense that lines of intermediate critical density and/or ionization potential have intermediate profile parameters.

We have adopted the area-defined parameters of Whittle (1982, 1985a) with a slight modification in the wings to allow for pixels that have negative flux after continuum subtraction. In Table 2 and Figures 8-13, we present correlations involving the Whittle parameters, critical density, and ionization potential. We have chosen the median velocity, $V(50 \%)$, and the interpercentile velocity width at $20 \%$. The later is defined in Whittle $(1982,1985 \mathrm{a})$ as IPV $(20 \%)=a+b$, where $a$ is the velocity (relative to the median) such that $10 \%$ of the total profile flux is blueward of $a$ (see Fig. 7). Analogously, $10 \%$ of the total profile flux is redward of $b$. To be consistent with other studies, we have included analysis and discussion of emission-line blueshift. However, the sensitivity of the blueshift to details of the obscuration mechanism, which is poorly constrained, complicates the interpretation (see also Moore 1994, 1995). Therefore, we emphasize the line widths, because their correspondence to NLR kinematics is more direct than the blueshifts.

For reference, we have also included in Table 2 the value of the IPV and asymmetry functions averaged between $20 \%$ and $80 \%\left(\mathrm{IPV}_{\text {avg }}\right.$ and $\left.A_{\text {avg }}\right)$, the asymmetry function at $20 \%$ $(A[20 \%])$, and the kurtosis of the profiles. The asymmetry function is defined in Whittle $(1982,1985 a)$ as

$$
A(\%)=\frac{a(\% / 2)-b(\% / 2)}{a(\% / 2)+b(\% / 2)}
$$

The asymmetry function is positive for blueward extension and negative for redward extension. The kurtosis is defined in Whittle $(1982,1985 \mathrm{a})$ as $2.5[\operatorname{IPV}(50 \%) / \operatorname{IPV}(10 \%)]$, where the normalization is chosen so that a Gaussian has kurtosis of 1 . Kurtosis less than 1 corresponds to a profile more cusplike than a Gaussian, and the converse is true for 
TABLE 2

Whittle Parameters

\begin{tabular}{|c|c|c|c|c|c|c|}
\hline Line & $\begin{array}{c}V(50 \%) \\
\left(\mathrm{km} \mathrm{s}^{-1}\right)\end{array}$ & $\begin{array}{c}\operatorname{IPV}(20 \%) \\
\left(\mathrm{km} \mathrm{s}^{-1}\right)\end{array}$ & $\begin{array}{c}\mathrm{IPV}_{\mathrm{avg}} \\
\left(\mathrm{km} \mathrm{s}^{-1}\right)\end{array}$ & Kurtosis & $A(20 \%)$ & $A_{\mathrm{av8}}$ \\
\hline \multicolumn{7}{|c|}{ MCG 8-11-11 } \\
\hline 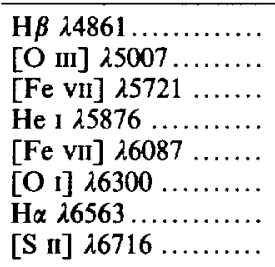 & $\begin{array}{r}124 \\
92 \\
16 \\
43 \\
-29 \\
1 \\
105 \\
78\end{array}$ & $\begin{array}{l}828 \\
775 \\
646 \\
665 \\
867 \\
871 \\
454 \\
695\end{array}$ & $\begin{array}{l}352 \\
340 \\
301 \\
308 \\
371 \\
356 \\
205 \\
299\end{array}$ & $\begin{array}{l}0.87 \\
0.90 \\
1.10 \\
1.05 \\
0.90 \\
0.80 \\
1.03 \\
0.91\end{array}$ & $\begin{array}{l}0.09 \\
0.07 \\
0.15 \\
0.20 \\
0.18 \\
0.12 \\
0.09 \\
0.08\end{array}$ & $\begin{array}{l}0.11 \\
0.06 \\
0.07 \\
0.11 \\
0.09 \\
0.05 \\
0.05 \\
0.03\end{array}$ \\
\hline \multicolumn{7}{|c|}{ Mrk 79} \\
\hline 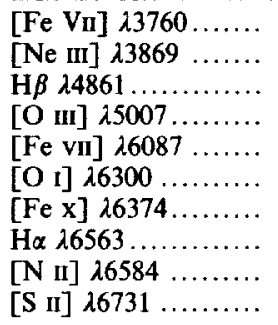 & $\begin{array}{r}-141 \\
53 \\
44 \\
61 \\
-30 \\
58 \\
-113 \\
35 \\
21 \\
19\end{array}$ & $\begin{array}{l}906 \\
440 \\
400 \\
493 \\
708 \\
403 \\
838 \\
379 \\
424 \\
313\end{array}$ & $\begin{array}{l}427 \\
190 \\
183 \\
205 \\
295 \\
175 \\
360 \\
170 \\
184 \\
142\end{array}$ & $\begin{array}{l}1.12 \\
0.87 \\
0.98 \\
0.69 \\
0.90 \\
0.84 \\
1.01 \\
1.00 \\
0.93 \\
1.06\end{array}$ & $\begin{array}{r}0.05 \\
0.14 \\
0.08 \\
-0.05 \\
0.30 \\
-0.04 \\
0.26 \\
0.00 \\
0.12 \\
0.16\end{array}$ & $\begin{array}{r}0.13 \\
0.05 \\
0.06 \\
-0.01 \\
0.15 \\
0.00 \\
0.08 \\
-0.01 \\
0.03 \\
0.08\end{array}$ \\
\hline \multicolumn{7}{|c|}{ Mrk 704} \\
\hline 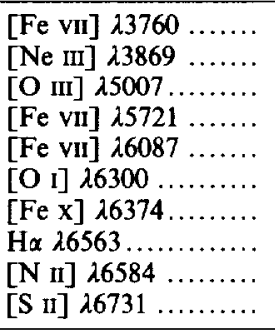 & $\begin{array}{r}-122 \\
31 \\
62 \\
-96 \\
-94 \\
69 \\
-102 \\
-1 \\
80 \\
52 \\
\end{array}$ & $\begin{array}{l}572 \\
449 \\
418 \\
412 \\
649 \\
294 \\
479 \\
338 \\
305 \\
340 \\
\end{array}$ & $\begin{array}{l}231 \\
192 \\
183 \\
168 \\
254 \\
136 \\
215 \\
154 \\
138 \\
154\end{array}$ & $\begin{array}{l}0.777 \\
0.866 \\
0.911 \\
0.834 \\
0.769 \\
1.13 \\
0.96 \\
1.05 \\
1.04 \\
1.08\end{array}$ & $\begin{array}{r}0.232 \\
0.002 \\
0.061 \\
0.236 \\
0.291 \\
0.037 \\
0.093 \\
-0.075 \\
0.030 \\
0.146 \\
\end{array}$ & $\begin{array}{r}0.143 \\
-0.028 \\
0.015 \\
0.114 \\
0.180 \\
0.018 \\
0.040 \\
-0.053 \\
0.024 \\
0.041\end{array}$ \\
\hline \multicolumn{7}{|c|}{ Mrk 841} \\
\hline 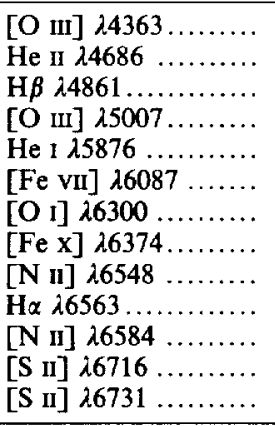 & $\begin{array}{r}78 \\
70 \\
51 \\
66 \\
53 \\
-138 \\
39 \\
-47 \\
48 \\
39 \\
32 \\
36 \\
21 \\
\end{array}$ & $\begin{array}{r}306 \\
268 \\
421 \\
306 \\
165 \\
1435 \\
193 \\
394 \\
139 \\
306 \\
280 \\
186 \\
201 \\
\end{array}$ & $\begin{array}{r}128 \\
114 \\
145 \\
119 \\
73 \\
543 \\
84 \\
181 \\
62 \\
117 \\
110 \\
80 \\
87\end{array}$ & $\begin{array}{l}0.89 \\
0.92 \\
0.69 \\
0.69 \\
1.02 \\
0.76 \\
0.94 \\
1.08 \\
1.02 \\
0.72 \\
0.78 \\
0.92 \\
0.89 \\
\end{array}$ & $\begin{array}{r}-0.04 \\
-0.02 \\
0.41 \\
0.06 \\
-0.02 \\
0.44 \\
-0.05 \\
0.20 \\
0.13 \\
0.12 \\
0.25 \\
0.07 \\
0.03 \\
\end{array}$ & $\begin{array}{r}-0.03 \\
-0.05 \\
0.15 \\
0.01 \\
0.00 \\
0.22 \\
-0.02 \\
0.17 \\
0.06 \\
0.04 \\
0.10 \\
0.02 \\
-0.02 \\
\end{array}$ \\
\hline \multicolumn{7}{|c|}{ NGC 4151} \\
\hline 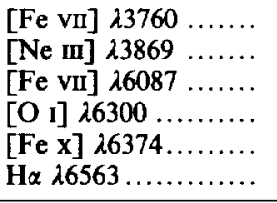 & $\begin{array}{r}-114 \\
-55 \\
-49 \\
36 \\
-156 \\
32 \\
\end{array}$ & $\begin{array}{r}721 \\
777 \\
791 \\
541 \\
1054 \\
381 \\
\end{array}$ & $\begin{array}{l}299 \\
292 \\
285 \\
208 \\
500 \\
155\end{array}$ & $\begin{array}{l}0.86 \\
0.71 \\
0.68 \\
0.74 \\
1.08 \\
0.86 \\
\end{array}$ & $\begin{array}{l}0.12 \\
0.19 \\
0.26 \\
0.05 \\
0.29 \\
0.03\end{array}$ & $\begin{array}{l}0.09 \\
0.11 \\
0.17 \\
0.04 \\
0.21 \\
0.02 \\
\end{array}$ \\
\hline \multicolumn{7}{|c|}{ NGC 5548} \\
\hline 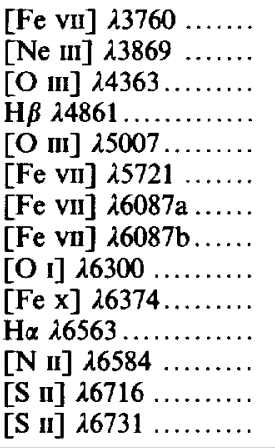 & $\begin{array}{r}-376 \\
-151 \\
-303 \\
-98 \\
-132 \\
-287 \\
-289 \\
-334 \\
-127 \\
-436 \\
-57 \\
31 \\
26 \\
19\end{array}$ & $\begin{array}{l}564 \\
574 \\
521 \\
491 \\
547 \\
572 \\
717 \\
726 \\
627 \\
762 \\
417 \\
283 \\
306 \\
327\end{array}$ & $\begin{array}{l}252 \\
259 \\
225 \\
224 \\
234 \\
262 \\
303 \\
302 \\
249 \\
304 \\
195 \\
126 \\
144 \\
149\end{array}$ & $\begin{array}{l}1.03 \\
0.97 \\
0.91 \\
1.05 \\
0.85 \\
0.99 \\
0.83 \\
0.89 \\
0.76 \\
0.82 \\
1.12 \\
1.01 \\
1.09 \\
1.04\end{array}$ & $\begin{array}{l}0.05 \\
0.04 \\
0.24 \\
0.08 \\
0.14 \\
0.00 \\
0.05 \\
0.10 \\
0.31 \\
0.17 \\
0.12 \\
0.13 \\
0.07 \\
0.07\end{array}$ & $\begin{array}{l}0.04 \\
0.03 \\
0.08 \\
0.05 \\
0.07 \\
0.02 \\
0.02 \\
0.02 \\
0.16 \\
0.00 \\
0.06 \\
0.05 \\
0.05 \\
0.02\end{array}$ \\
\hline
\end{tabular}



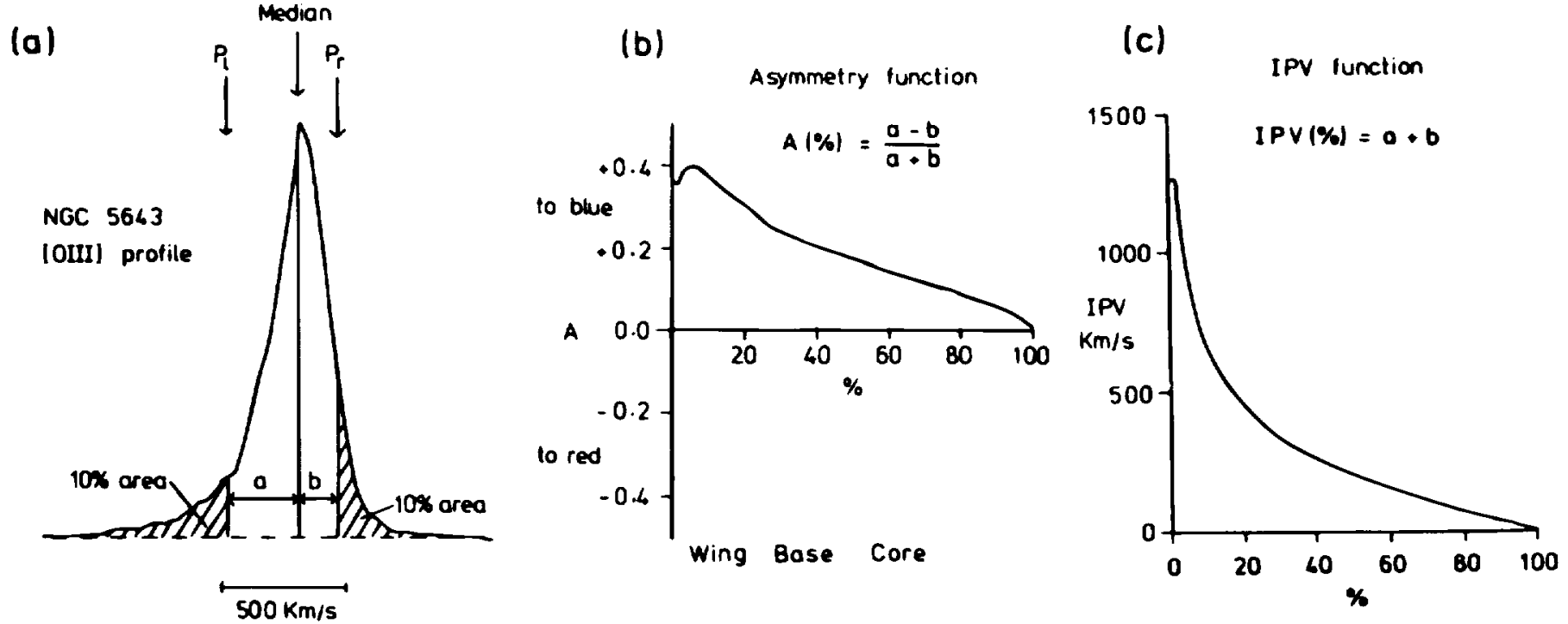

FIG. 7.-Definitions for an area parameter scheme to characterize profile asymmetry and width. (a) 10,50 , and 90 interpercentile markers in the [O III] profile of NGC 5643 (wavelength increasing to the right). Lengths $a$ and $b$ are used to evaluate asymmetry and width parameters $A(20 \%)$ and IPV(20\%), respectively. $(b, c)$ Asymmetry and IPV functions for this profile. This figure and caption are both reproduced from Whittle (1985a). We thank Mark Whittle, as well as the MNRAS, for permission to include them here.
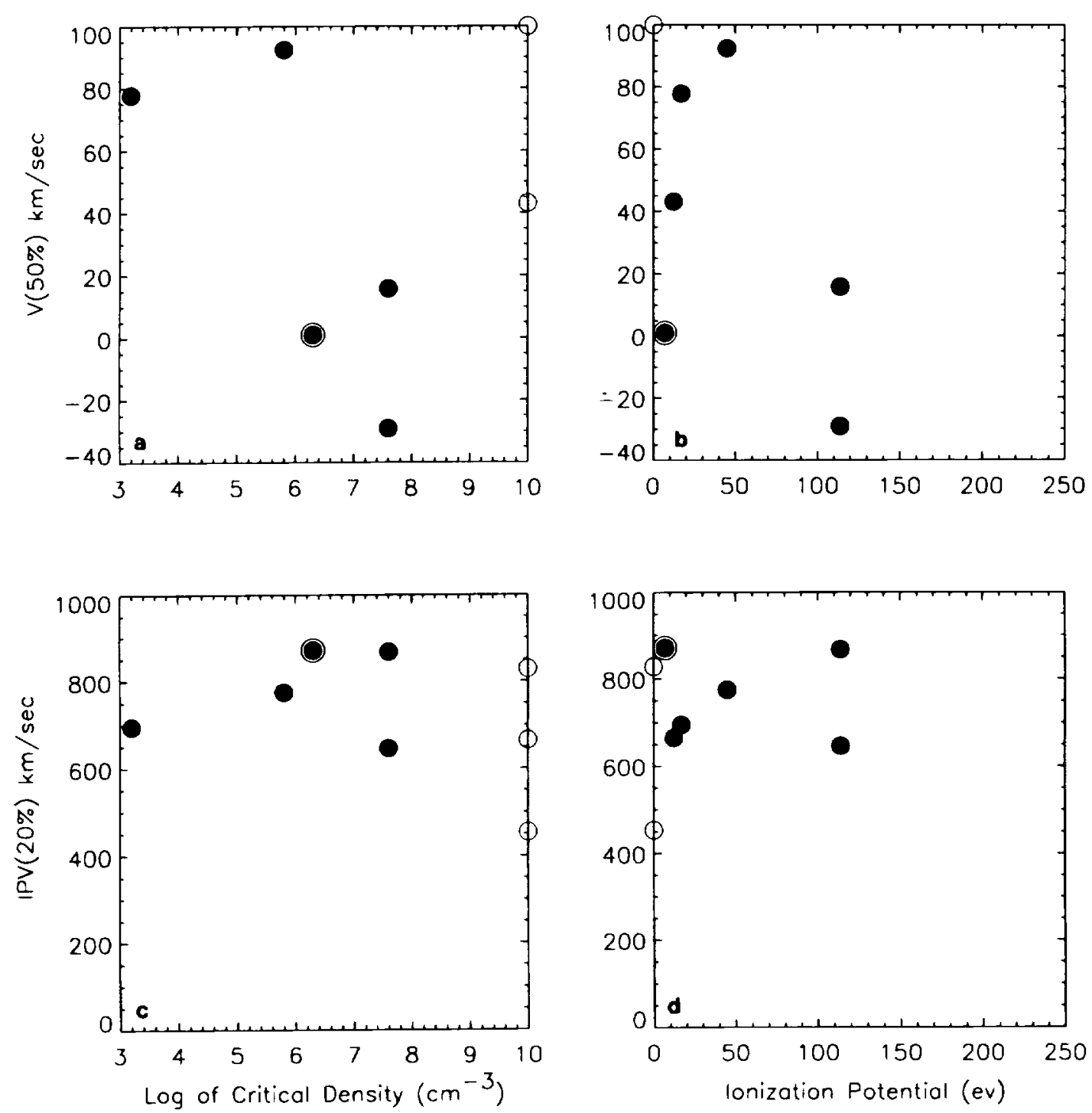

FIG. 8. - The median velocity, $V(50 \%)$, and the interpercentile velocity width at $20 \%$ (see text and Fig. 7 ) are plotted vs. $n_{\mathrm{cr}}$ and IP for MCG $8-11-11$. Isolated open circles represent the recombination lines, and the open circle enclosing the filled circle represents [O $\mathrm{t}] \lambda 6300$. There is no correlation of $\operatorname{IPV}(20 \%)$ with either $n_{\mathrm{cr}}$ or IP. 

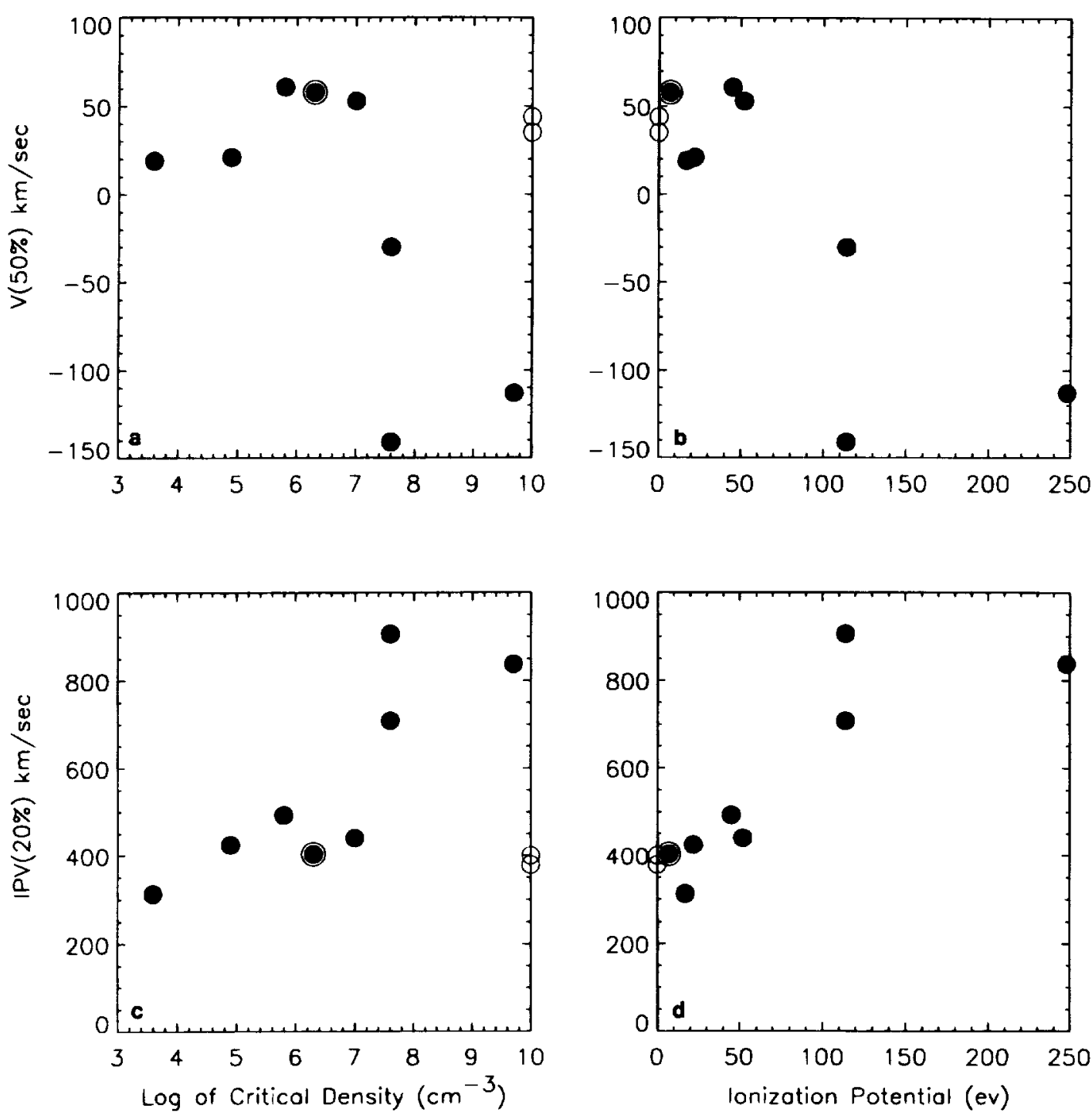

FiG. 9. - The median velocity, $V(50 \%)$, and the interpercentile velocity width at $20 \%$ (see text) are plotted vs. $n_{c r}$ and IP for Mrk 79 . Isolated open circles represent the recombination lines. Although IPV $(20 \%)$ correlates with both $n_{\mathrm{cr}}$ and IP, [O I] $\lambda 6300$ (enclosed by an open circle) lies off the correlation on the $n_{\mathrm{cr}}$ plot, but it follows the correlation on the IP plot.

kurtosis greater than 1 . The values of $V(50 \%)$, IPV $(20 \%)$, and IPV $_{\mathrm{avg}}$ are in units of $\mathrm{km} \mathrm{s}^{-1}$, while the kurtosis, $A(20 \%)$, and $A_{\mathrm{avg}}$ are dimensionless.

For possible future reference, we present six additional profile parameters in Table 3. The velocity of the peak and centroid are $V_{\text {peak }}$ and $V_{\text {centroid. }}$. The remaining parameters are widths as follows. The rms velocity is $V_{\mathrm{rms}} \equiv\left(\left\langle v^{2}\right\rangle\right.$ $\left.-\langle v\rangle^{2}\right)^{1 / 2}$. The full widths at $\frac{1}{4}, \frac{1}{3}$, and $\frac{1}{2}$ of the peak intensity are $F W \frac{1}{4} M, F W \frac{1}{3} M$, and FWHM, respectively. All the parameters of Table 3 are in units of $\mathrm{km} \mathrm{s}^{-1}$.

As an additional reference, we have also tabulated those emission lines we have analyzed together with their critical density and ionization potential in Table 4 . As is conventional, we define the ionization potential as the arithmetic mean of the two successive stages of ionization.

In Figures 8-13, we plot $V(50 \%)$ and IPV $(20 \%)$ in units of $\mathrm{km} \mathrm{s}^{-1}$ versus the $\log$ of $n_{\mathrm{cr}}$ in units $\mathrm{cm}^{-3}$ and versus IP in $\mathrm{eV}$ for all six of our objects. Because much of the discussion that follows focuses on [O I] $\lambda 6300$ and [O III] $\lambda 4363$ as the lines that can potentially discriminate between correlations with $n_{\mathrm{cr}}$ and correlations with IP, we highlight the data points corresponding to these lines by enclosing them with open circles. The isolated open circles represent $\mathrm{H} \alpha$ and $\mathrm{H} \beta$. Although the critical density and ionization potential are not physically meaningful for the recombination lines, the profiles contain information. In stratified clouds, the recombination lines are emitted most efficiently in a zone of intermediate ionization, in which [O III] and [Ne III] are also emitted. Due to the relatively large uncertainties of subtracting the underlying broad component from the narrow component, it is not appropriate here to attach much significance to the similar velocity shifts (and line widths) of the Balmer lines and the lines of [O III] $\lambda 5007$ and $[\mathrm{Ne}$ III] $\lambda 3869$. However, we suggest that the recombination lines should be included in similar analyses of Seyfert 2 galaxies.

For NGC 5548, correlations of both $V(50 \%)$ and IPV $(20 \%)$ are clearly with $n_{\mathrm{cr}}$ rather than with IP, making this object a counterexample to the general trend discussed here and in MC. Therefore, we discuss the correlations for NGC 5548 separately. For the other objects, correlations are less clear, particularly the correlation with $V(50 \%)$. 

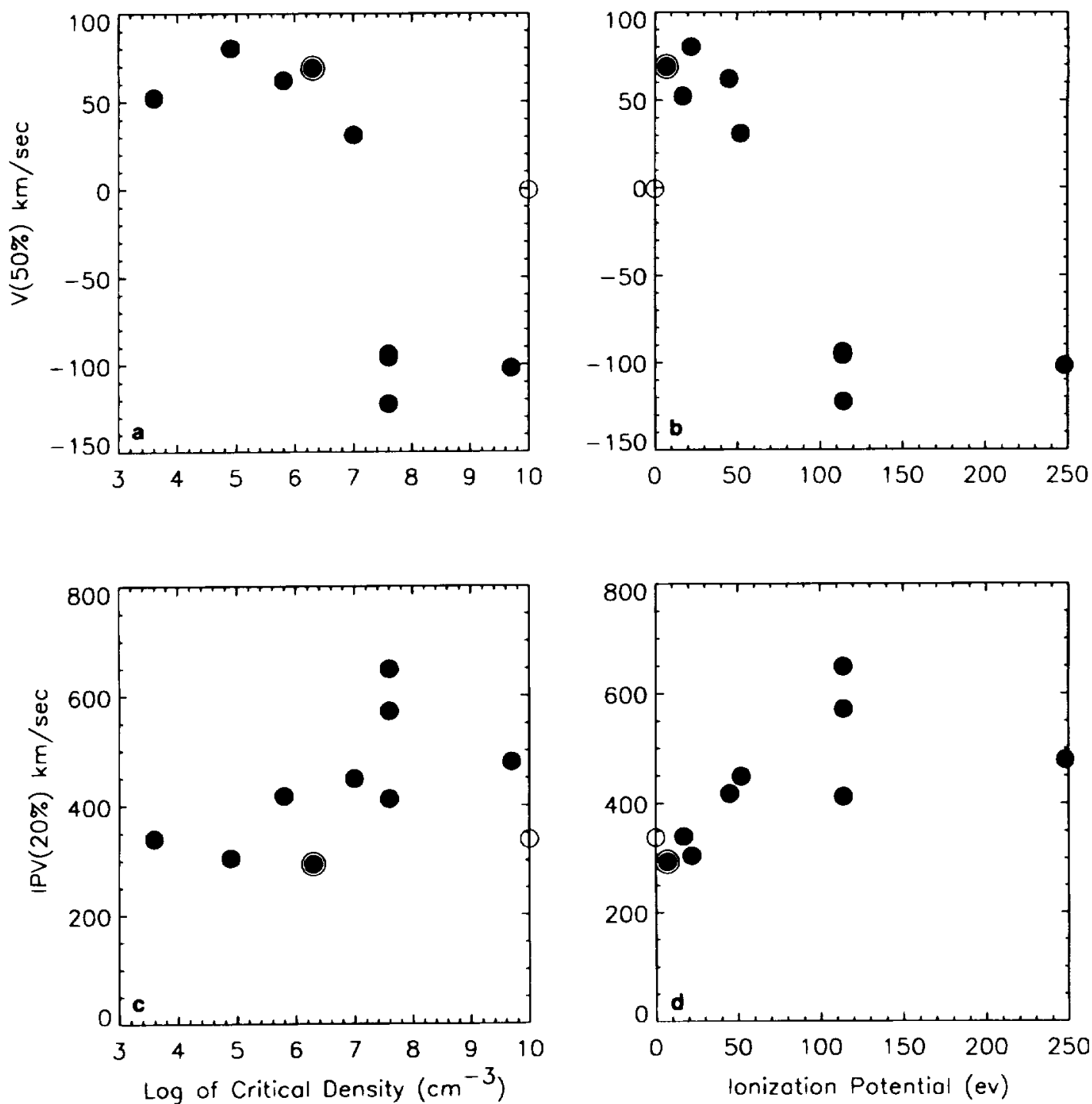

FIG. 10 .-The median velocity, $V(50 \%)$, and the interpercentile velocity width at $20 \%$ (see text) are plotted vs. $n_{\mathrm{cr}}$ and IP for Mrk 704 . Isolated open circles represent the recombination lines. Although IPV $(20 \%)$ correlates with both $n_{c r}$ and IP, [O I] $\lambda 6300$ (enclosed by an open circle) lies off the correlation on the $n_{\mathrm{cr}}$ plot but follows the correlation on the IP plot.

Ultimately, possible correlations between $V(50 \%)$ and/or IPV $(20 \%)$ with $n_{\mathrm{cr}}$ and/or IP must be accepted or rejected by comparing visually the scatter in the result with some systematic trend or lack thereof. Error bars that truly reflect a quantitative uncertainty in the parameters in cases in which nonformal errors dominate, such as that of [Fe $\mathrm{x}]$ $\lambda 6374$, are virtually impossible to estimate reliably. Only in the case in which the adjacent continuum is nearly flat or linear, and featureless, and in which there are no blends with other lines, are the true uncertainties approximated reasonably well by formal error estimates.

We estimated formal errors by choosing the $[\mathrm{O} \mathrm{III}] \lambda 5007$ profile in NGC 5548 as a fiducial model profile. We added a linear continuum and noise to the profile, subtracted a linear fit to the continuum, and calculated $V(50 \%)$ and IPV $(20 \%)$ for the resulting profile. We repeated the procedure 10 times and calculated the standard deviation in the 10 calculated values of $V(50 \%)$ and $\operatorname{IPV}(20 \%)$. We repeated the experiment with varying amounts of noise and continuum strength and slope. For fixed $S / N$ in the final continuum-subtracted profile, the standard deviations are not very sensitive to either the strength or the slope of the synthetic continuum. The reason is that we know a priori the functional form of the adjacent continuum, though this, of course, is not the case for the real data. The standard deviations are also not very sensitive to the precise choice of profile limits, though this again is at least partly an artifact of the continuum subtraction being more reliable than it is for the real data.

Letting $f$ be the noise added to the profile relative to the peak of the profile, the standard deviation in $V(50 \%)$ increases linearly from 0 to $70 \mathrm{~km} \mathrm{~s}^{-1}$ as $f$ is increased from 0 to 0.3 . For $\operatorname{IPV}(20 \%)$, the standard deviation increases linearly from 0 to $230 \mathrm{~km} \mathrm{~s}^{-1}$. (The standard deviation curves are more complicated for $f \gtrsim 0.3$.) The noisiest profiles that we analyzed have $f \leqslant 0.1$. It follows that the scatter in the following plots that do not show correlations (as well as those that do) is due mostly to either nonformal errors or to the likelihood that the objects are more complicated than justifies assuming an intrinsic one-to-one correspondence between line width and $n_{\mathrm{cr}}$ and/or IP. In Paper II, for example, we present synthetic correlations resulting from 

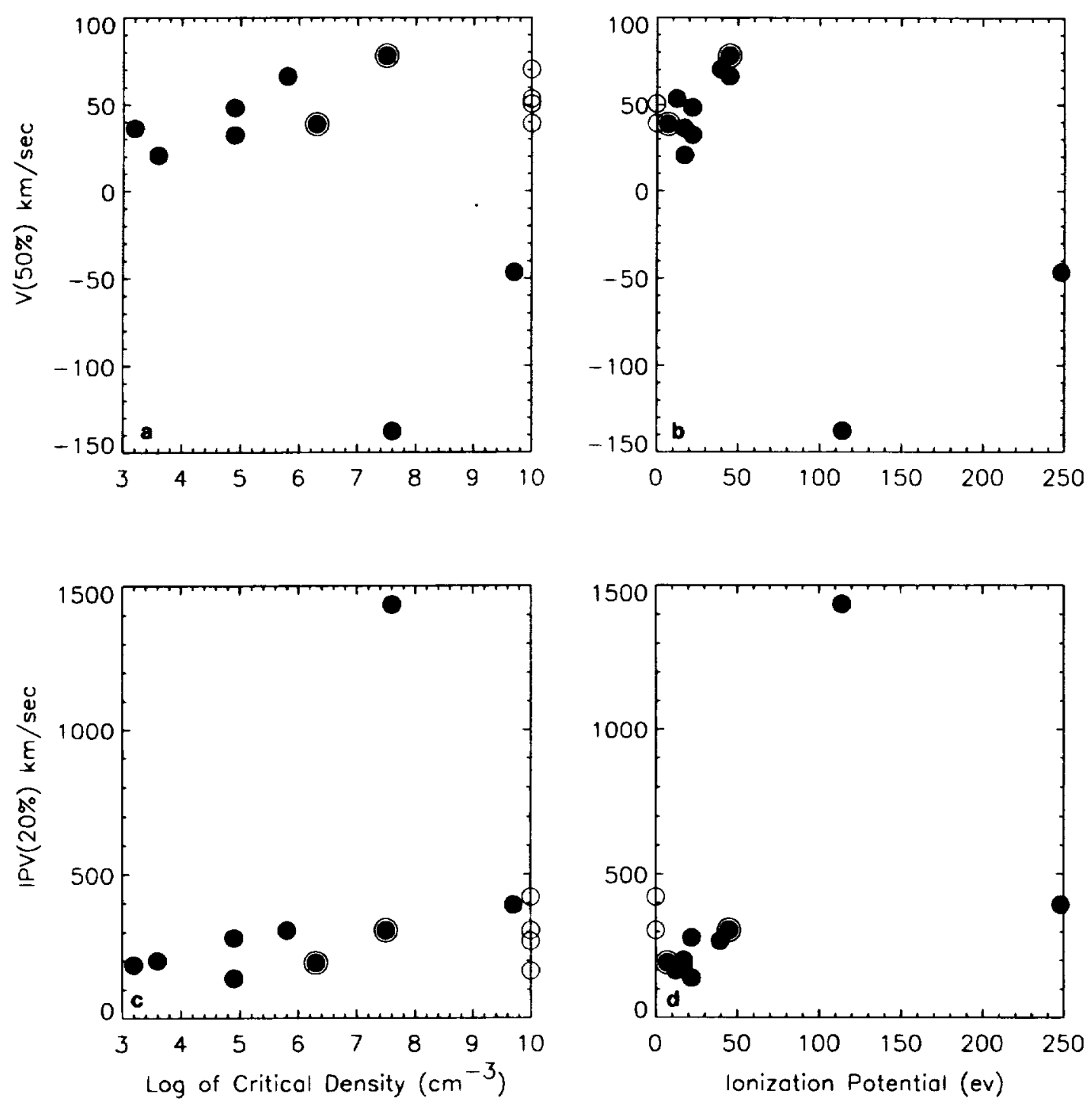

FIG. 11. - The median velocity, $V(50 \%)$, and the interpercentile velocity width at $20 \%$ (see text) are plotted vs. $n_{\text {cr }}$ and IP for Mrk 841 . Isolated open circles represent the recombination lines, and open circles enclosing the filled circles represent $\left[\mathrm{O}_{1}\right] \lambda 6300$ and $[\mathrm{O}$ III] $\lambda 4363$. It is inconclusive whether correlations are with IP or $n_{\mathrm{cr}}$ because only the [Fe] lines, which have both high $n_{\mathrm{cr}}$ and high IP, stand out.

models, in which scatter comparable to that observed is intrinsic to the model.

Now we present and discuss the correlations for individual objects. For each correlation, we also calculate a linear correlation coefficient, $R$, together with the corresponding probability of chance occurance, $P_{\text {null }}$.

MCG 8-11-11 (Fig. 8).-V(50\%) appears to correlate better with $n_{\text {cr }}$ than with IP. However, because [O III] $\lambda 5007$ is not blueshifted relative to [S II] $\lambda 6716$, any correlation between blueshift and critical density that might be inferred from Figure $8 a$ is at best tenuous. There is no correlation of IPV $(20 \%)$ with either $n_{\text {cr }}$ or IP (we have already noted in $\S 5.1$ that the widths, as well as the profiles, of lines of both high and low IP are similar in this object). For MCG 8-11$11, R / P_{\text {null }}$ are $-0.73 / 0.16$ for $V(50 \%)$ versus $n_{\mathrm{cr}},-0.50 /$ 0.39 for $V(50 \%)$ versus IP, $0.27 / 0.66$ for IPV $(20 \%)$ versus $n_{\mathrm{cr}}$, and $-0.18 / 0.78$ for IPV $(20 \%)$ versus IP, respectively.

Mrk 79 (Fig. 9).-It appears that the highest critical density lines are blueshifted relative to the low critical density lines (Fig. 9a). Note, however, that these lines have high IP and that [ $\left.\mathrm{O}_{1}\right] \lambda 6300$ is associated with the lines of low-ionization potential rather than with those of high critical density. Although a correlation with $n_{\text {cr }}$ is apparent visually, to interpret Figure $9 a$ as a correlation of $V(50 \%)$ with $n_{\mathrm{cr}}$, we must suppose that $V(50 \%)$ is roughly constant over four decades in critical density and then changes rapidly over an additional factor of order unity in $n_{\mathrm{cr}}$. For IPV $(20 \%), \lambda 6300$ lies off the correlation on the critical density plot (Fig. 9c) but follows the correlation on the ionization potential plot (Fig. $9 d$ ). The values of $R / P_{\text {null }}$ are $-0.64 / 0.08$ for $V(50 \%)$ versus $n_{\text {cr }},-0.79 / 0.02$ for $V(50 \%)$ versus IP, $0.83 / 0.02$ for IPV $(20 \%)$ versus $n_{\mathrm{cr}}$, and $0.84 / 0.01$ for IPV( $20 \%)$ versus IP, respectively.

Mrk 704 (Fig. 10).-On the $V(50 \%)$ correlation, the [O I] line fits on both the $n_{\text {cr }}$ and the IP relations. On the IPV plot, the $\left[\mathrm{O}_{\mathrm{I}}\right]$ line fits well on the IP correlation and falls out of place on the $n_{\mathrm{cr}}$ correlation. The values of $R / P_{\text {null }}$ are $-0.79 / 0.01,0.61 / 0.08,-0.82 / 0.006$, and $0.58 / 0.10$.

Mrk 841 (Fig. 11). - For all the correlations for Mrk 841, only the $[\mathrm{Fe}$ vII] and $[\mathrm{Fe} \mathrm{x}]$ lines stand out. It is inconclusive whether correlations are with IP or with $n_{\mathrm{cr}}$ because these lines have both high IP and high $n_{\mathrm{cr}}$. The values of $R / P_{\text {null }}$ are $-0.46 / 0.22,-0.62 / 0.07,0.44 / 0.24$, and $0.40 / 0.28$. 
TABLE 3

additional Profile Parameters

\begin{tabular}{|c|c|c|c|c|c|c|}
\hline Line & $V_{\text {peak }}$ & $V_{\text {centroid }}$ & $V_{\mathrm{rms}}$ & $F W \frac{1}{4} M$ & $\mathrm{FW} \frac{1}{3} \mathrm{M}$ & FWHM \\
\hline \multicolumn{7}{|c|}{ MCG 8-11-11 } \\
\hline 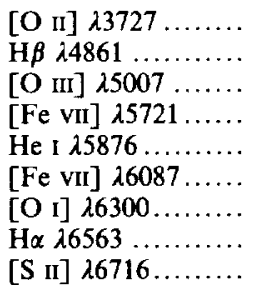 & $\begin{array}{r}71 \\
115 \\
119 \\
126 \\
83 \\
136 \\
117 \\
121 \\
64\end{array}$ & $\begin{array}{r}-46 \\
48 \\
19 \\
-50 \\
-26 \\
-106 \\
-74 \\
56 \\
30\end{array}$ & $\begin{array}{l}395 \\
331 \\
323 \\
279 \\
288 \\
351 \\
390 \\
197 \\
287\end{array}$ & $\begin{array}{r}1134 \\
888 \\
899 \\
895 \\
810 \\
955 \\
912 \\
611 \\
1185\end{array}$ & $\begin{array}{r}1011 \\
764 \\
757 \\
811 \\
748 \\
846 \\
755 \\
544 \\
690\end{array}$ & $\begin{array}{l}823 \\
544 \\
624 \\
607 \\
650 \\
606 \\
577 \\
413 \\
555\end{array}$ \\
\hline \multicolumn{7}{|c|}{ Mrk 79} \\
\hline 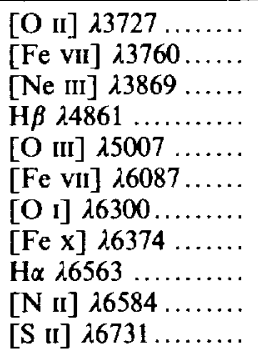 & $\begin{array}{r}23 \\
-160 \\
-12 \\
18 \\
-7 \\
-4 \\
25 \\
-31 \\
-8 \\
-16 \\
13\end{array}$ & $\begin{array}{r}-46 \\
-249 \\
-49 \\
-30 \\
25 \\
-119 \\
53 \\
-201 \\
3 \\
-27 \\
-32\end{array}$ & $\begin{array}{l}201 \\
318 \\
179 \\
159 \\
260 \\
316 \\
241 \\
306 \\
171 \\
195 \\
132\end{array}$ & $\begin{array}{r}725 \\
1009 \\
528 \\
509 \\
506 \\
733 \\
456 \\
906 \\
458 \\
471 \\
409\end{array}$ & $\begin{array}{r}658 \\
1003 \\
464 \\
451 \\
452 \\
593 \\
400 \\
772 \\
422 \\
425 \\
383\end{array}$ & $\begin{array}{l}550 \\
854 \\
433 \\
367 \\
425 \\
415 \\
353 \\
570 \\
342 \\
344 \\
313\end{array}$ \\
\hline \multicolumn{7}{|c|}{ Mrk 704} \\
\hline 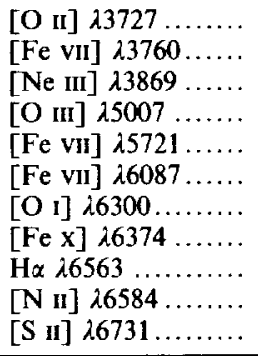 & $\begin{array}{r}92 \\
-175 \\
-70 \\
0 \\
-103 \\
-94 \\
42 \\
-116 \\
-67 \\
60 \\
104\end{array}$ & $\begin{array}{r}43 \\
-257 \\
-50 \\
-12 \\
-158 \\
-181 \\
30 \\
-155 \\
-29 \\
42 \\
26 \\
\end{array}$ & $\begin{array}{l}179 \\
190 \\
183 \\
161 \\
185 \\
303 \\
256 \\
217 \\
148 \\
135 \\
137\end{array}$ & $\begin{array}{l}737 \\
627 \\
512 \\
476 \\
483 \\
511 \\
416 \\
544 \\
457 \\
405 \\
473\end{array}$ & $\begin{array}{l}597 \\
402 \\
446 \\
429 \\
315 \\
410 \\
379 \\
576 \\
401 \\
365 \\
439\end{array}$ & $\begin{array}{l}448 \\
298 \\
362 \\
393 \\
233 \\
262 \\
315 \\
460 \\
318 \\
292 \\
305\end{array}$ \\
\hline \multicolumn{7}{|c|}{ Mrk 841} \\
\hline 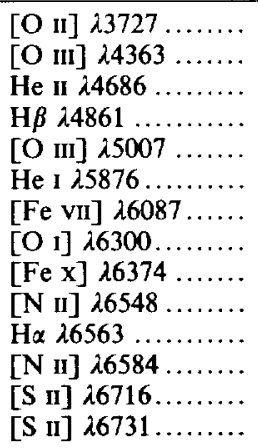 & $\begin{array}{r}-55 \\
-3 \\
-12 \\
4 \\
0 \\
14 \\
3 \\
0 \\
-58 \\
21 \\
9 \\
10 \\
4 \\
1\end{array}$ & $\begin{array}{r}31 \\
7 \\
13 \\
-53 \\
-2 \\
20 \\
-351 \\
6 \\
-112 \\
12 \\
-5 \\
-20 \\
-2 \\
4\end{array}$ & $\begin{array}{r}203 \\
112 \\
102 \\
155 \\
153 \\
71 \\
588 \\
90 \\
160 \\
61 \\
148 \\
127 \\
87 \\
89\end{array}$ & $\begin{array}{r}552 \\
394 \\
351 \\
300 \\
296 \\
246 \\
1337 \\
233 \\
491 \\
197 \\
267 \\
258 \\
224 \\
224\end{array}$ & $\begin{array}{l}515 \\
347 \\
280 \\
283 \\
258 \\
197 \\
718 \\
204 \\
438 \\
169 \\
231 \\
216 \\
192 \\
203\end{array}$ & $\begin{array}{l}445 \\
218 \\
206 \\
212 \\
189 \\
148 \\
639 \\
166 \\
313 \\
128 \\
171 \\
164 \\
150 \\
167\end{array}$ \\
\hline \multicolumn{7}{|c|}{ NGC 4151} \\
\hline 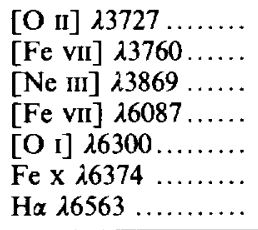 & $\begin{array}{r}-137 \\
-105 \\
-107 \\
-2 \\
4 \\
-2 \\
-1\end{array}$ & $\begin{array}{r}-116 \\
-217 \\
-107 \\
-89 \\
-33 \\
-233 \\
-7\end{array}$ & $\begin{array}{l}301 \\
280 \\
320 \\
372 \\
231 \\
437 \\
166\end{array}$ & $\begin{array}{r}795 \\
988 \\
644 \\
520 \\
500 \\
1092 \\
443 \\
\end{array}$ & $\begin{array}{l}723 \\
907 \\
511 \\
390 \\
391 \\
995 \\
353 \\
\end{array}$ & $\begin{array}{l}543 \\
678 \\
370 \\
283 \\
245 \\
543 \\
240 \\
\end{array}$ \\
\hline \multicolumn{7}{|c|}{ NGC 548} \\
\hline 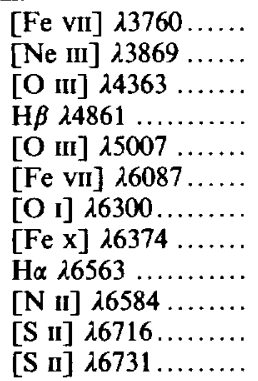 & $\begin{array}{r}-430 \\
-149 \\
-325 \\
-129 \\
-164 \\
-325 \\
-120 \\
-556 \\
-65 \\
33 \\
17 \\
-19\end{array}$ & $\begin{array}{l}-430 \\
-243 \\
-387 \\
-174 \\
-227 \\
-323 \\
-219 \\
-494 \\
-105 \\
-12 \\
-11 \\
-21\end{array}$ & $\begin{array}{l}224 \\
236 \\
224 \\
182 \\
248 \\
324 \\
275 \\
301 \\
179 \\
121 \\
127 \\
131\end{array}$ & $\begin{array}{r}1478 \\
682 \\
677 \\
621 \\
605 \\
790 \\
542 \\
777 \\
560 \\
378 \\
407 \\
422\end{array}$ & $\begin{array}{r}1141 \\
610 \\
573 \\
588 \\
538 \\
675 \\
464 \\
633 \\
517 \\
323 \\
366 \\
393\end{array}$ & $\begin{array}{l}490 \\
507 \\
470 \\
484 \\
411 \\
545 \\
329 \\
500 \\
424 \\
243 \\
300 \\
307\end{array}$ \\
\hline
\end{tabular}


TABLE 4

EMISSION LINES, $\lambda, n_{\mathrm{cr}}$, AND IP

\begin{tabular}{|c|c|c|c|}
\hline $\begin{array}{l}\text { Wavelength } \\
(\AA)\end{array}$ & Ion & $\begin{array}{c}n_{\mathrm{cr}} \\
\left(\mathrm{cm}^{-3}\right)\end{array}$ & $\begin{array}{c}\text { Mean Ionization } \\
\text { Potential } \\
(\mathrm{eV})\end{array}$ \\
\hline 3726.1 . & {$\left[\begin{array}{ll}\mathrm{O} & \mathrm{II}\end{array}\right]$} & $3.2 \times 10^{3}$ & 24 \\
\hline 3728.8 . & {$\left[\begin{array}{lll}0 & 11\end{array}\right]$} & $6.3 \times 10^{2}$ & 24 \\
\hline $3760.3 \ldots \ldots$ & {$[\mathrm{Fe} \mathrm{VH}]$} & $4.0 \times 10^{7}$ & 114 \\
\hline $3868.8 \ldots \ldots$ & [Ne III] & $1.0 \times 10^{7}$ & 52 \\
\hline $4363.2 \ldots \ldots$ & [O III] & $3.2 \times 10^{7}$ & 45 \\
\hline $4685.7 \ldots \ldots$ & He II & $\ldots$ & 40 \\
\hline $4861.3 \ldots \ldots$ & $\mathrm{H}_{\mathrm{I}}$ & & $\ldots$ \\
\hline $5006.8 \ldots \ldots$ & {$[\mathrm{O} \mathrm{III}]$} & $6.3 \times 10^{5}$ & $\dddot{45}$ \\
\hline $5721.1 \ldots$ & [Fe VII] & $4.0 \times 10^{7}$ & 114 \\
\hline 5875.7 . & He I & & 12 \\
\hline 6086.9 . & {$[\mathrm{Fe}$ VII $]$} & $4.0 \times 10^{7}$ & 114 \\
\hline $6300.3 \ldots \ldots$ & {$\left[\begin{array}{ll}\mathrm{O}_{1} & 1\end{array}\right]$} & $2.0 \times 10^{6}$ & 7 \\
\hline $6374.6 \ldots \ldots$ & {$[\mathrm{Fe} \mathrm{x}]$} & $5.0 \times 10^{9}$ & 248 \\
\hline $6548.1 \ldots \ldots$ & {$[\mathrm{N} \mathrm{nI}]$} & $7.9 \times 10^{4}$ & 22 \\
\hline $6562.8 \ldots \ldots$ & $\mathrm{H}_{\mathbf{I}}$ & & $\ldots$ \\
\hline $6583.4 \ldots \ldots$ & {$[\mathrm{N}$ il] } & $7.9 \times 10^{4}$ & 22 \\
\hline $6716.4 \ldots \ldots$ & {$[\mathrm{S}$ II] } & $1.6 \times 10^{3}$ & 17 \\
\hline $6730.8 \ldots \ldots$ & {$\left[\begin{array}{ll}S & \text { III }\end{array}\right]$} & $4.0 \times 10^{3}$ & 17 \\
\hline
\end{tabular}
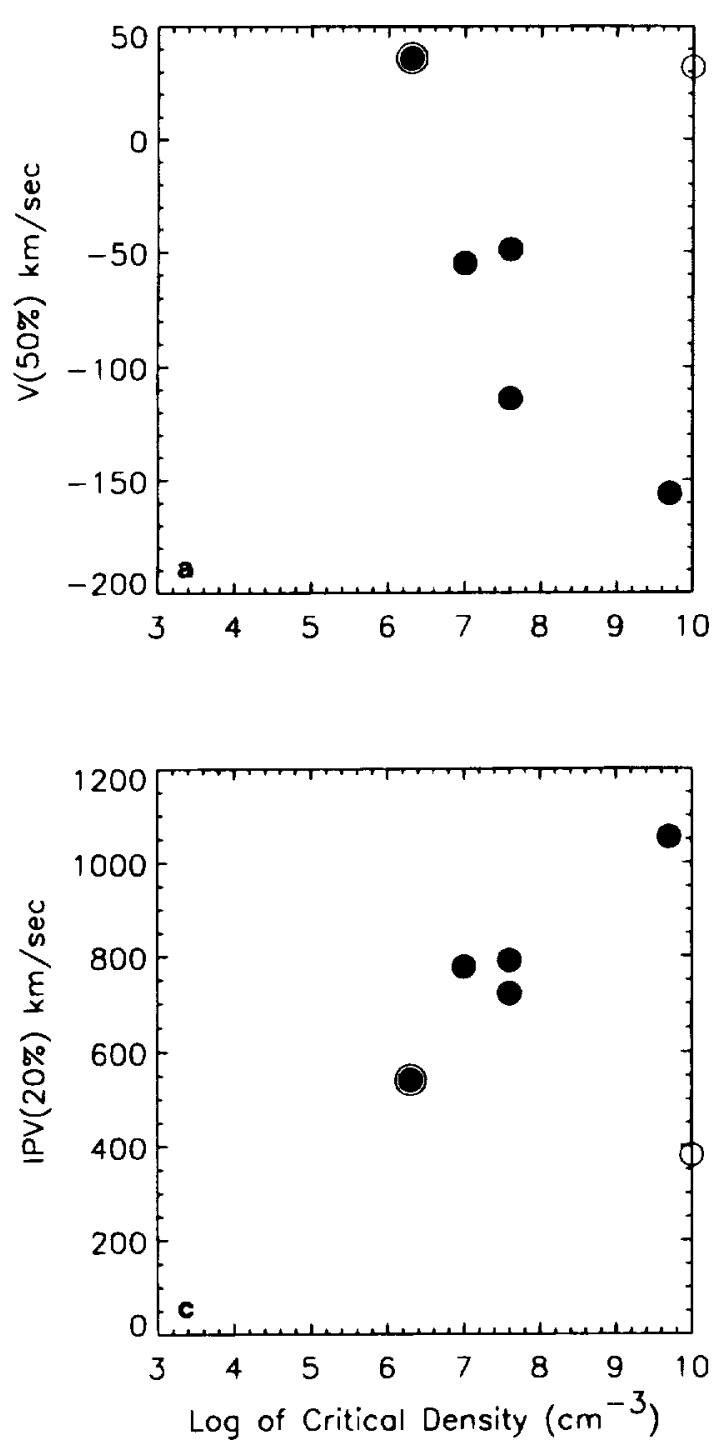

NGC 4151 (Fig. 12).-Because we do not have [S II] $\lambda 6716,6731$ profiles from NGC 4151 (and [O II] $\lambda 3727$ is an unresolved blend), all we can say about this object, based on our data alone, is that the highest IP $/ n_{\mathrm{cr}}$ lines are blueshifted and broad relative to [O I] $\lambda 6300$. However, if we were to include the [S II] profiles of Veilleux (1991a), we would put NGC 4151 in the same category as MCG 8-1111 (no correlation of IPV with either ionization potential or critical density).

NGC 5548 (Fig. 13).-Because the [O I] profile in NGC 5548 is broad and that of $[\mathrm{S} \mathrm{II}]$ is narrow, NGC 5548 stands in striking contrast to the general trend discussed here and in MC. In Figures $13 a$ and $13 b$, we plot the median velocity $(V[50 \%])$ of the emission lines from NGC 5548 versus both the critical density and the ionization potential, respectively. The velocities are relative to a $21 \mathrm{~cm}$ measurement of the systemic velocity.

As stated already, for NGC 5548, correlations of both $V(50 \%)$ and IPV $(20 \%)$ are clearly with $n_{\text {er }}$ rather than with IP. There is a good correlation between blueshift and criti-
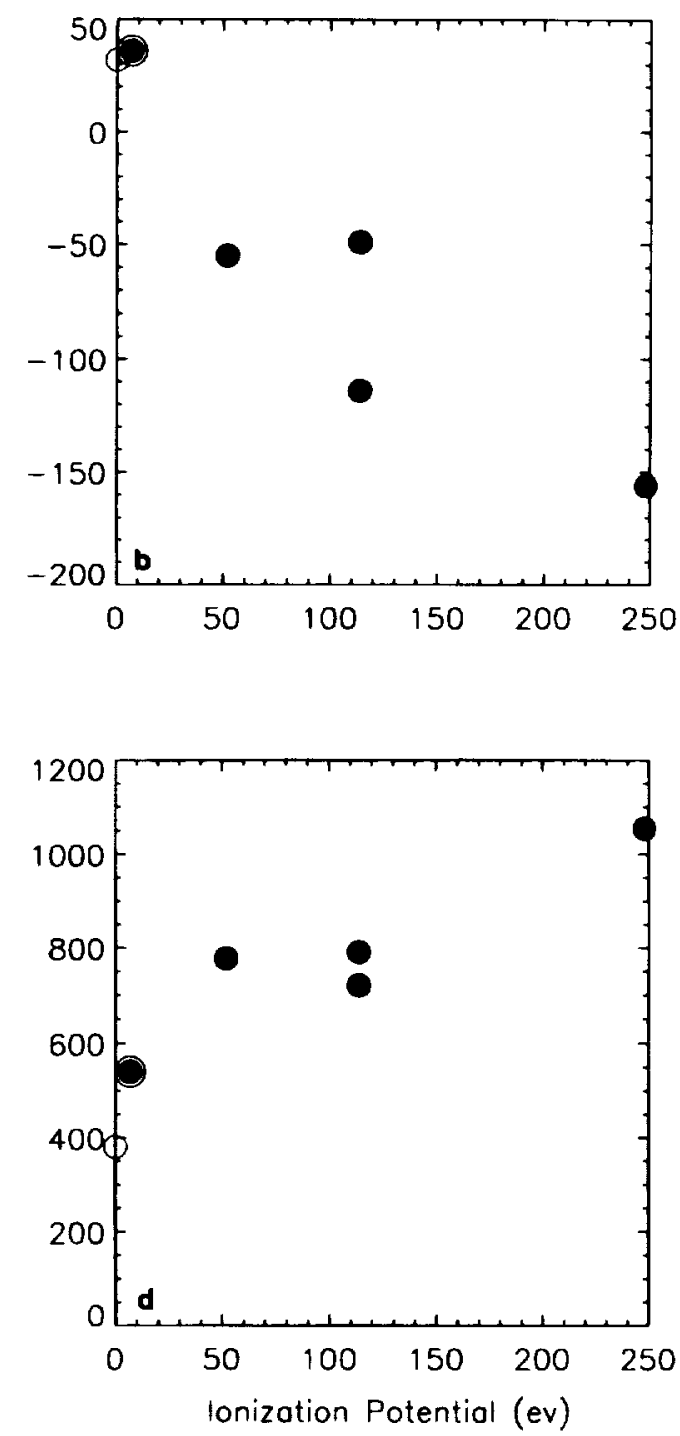

FIG. 12. - The median velocity, $V(50 \%)$, and the interpercentile velocity width at $20 \%$ (see text) are plotted vs. $n_{\text {cr }}$ and IP for NGC 4151 . Isolated open circle represents $\mathrm{H} \alpha$, and open circle enclosing the filled circle represents [O I] $\lambda 6300$. If we were to include the [S $\mathrm{n}$ ] $\lambda \lambda 6716,6731$ lines of Veilleux (1991a), we would infer no correlation of IPV $(20 \%)$ with either $n_{\mathrm{cr}}$ or IP. 

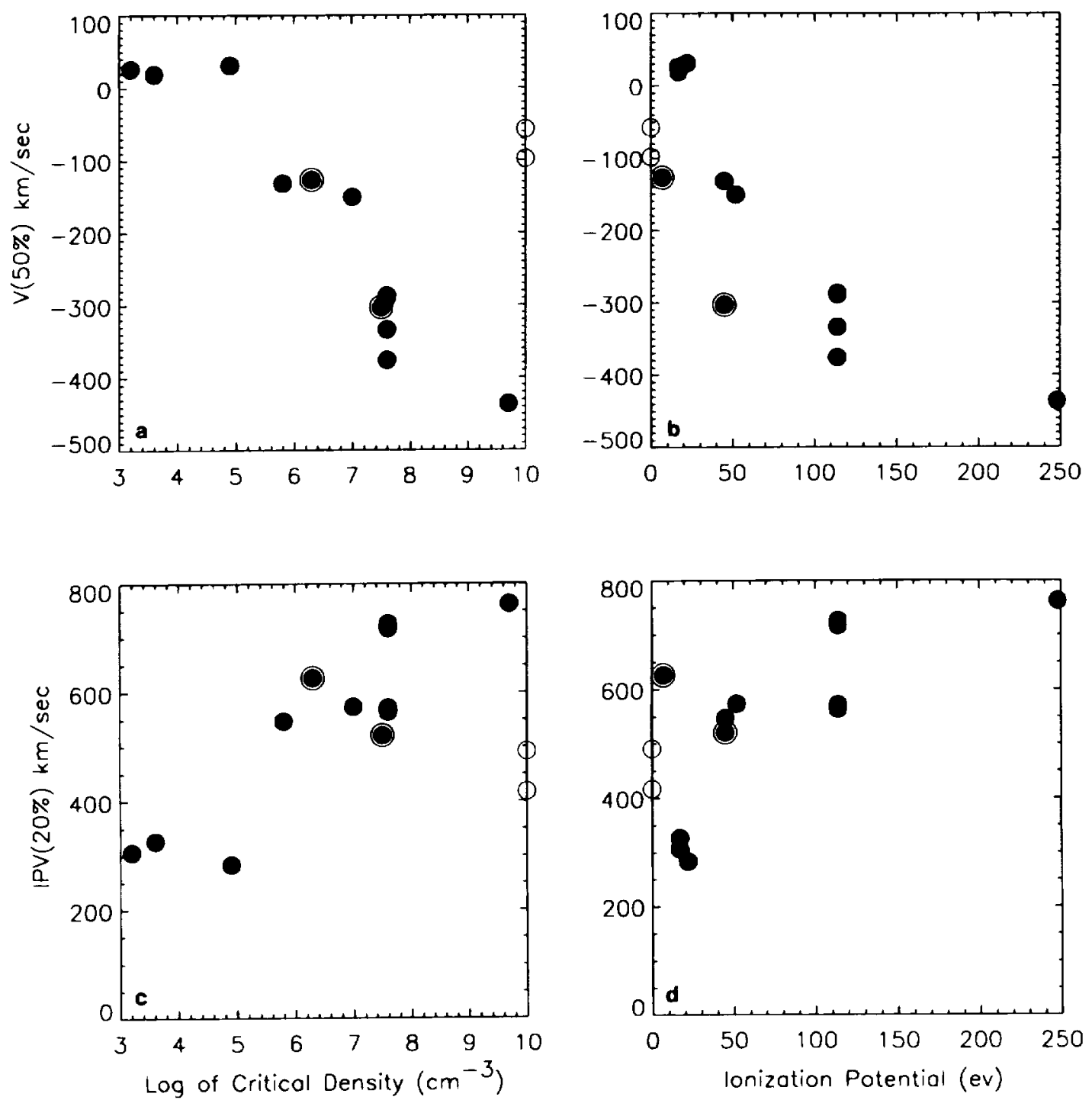

FIG. 13.-The median velocity, $V(50 \%)$, and the interpercentile velocity width at $20 \%$ (see text) are plotted vs. $n_{\mathrm{cr}}$ and IP for NGC 5548 . Isolated open circles represent the recombination lines. The important [ $\left[\mathrm{O}_{1}\right] \lambda 6300$ and $[\mathrm{O} \mathrm{III}] \lambda 4363$ data points are enclosed by open circles. Correlations of both $V(50 \%)$ and IPV $(20 \%)$ are clearly with $n_{\mathrm{cr}}$ rather than with IP. This is in contrast to the general trend discussed here and in MC.

cal density in NGC 5548. Although there is apparently a good correlation between blueshift and ionization potential as well, there are two points on the $V(50 \%)$ versus ionization potential plot that deviate. That these deviant points represent $\left[\mathrm{O}_{\mathrm{I}}\right] \lambda 6300$ and [O $\left.\mathrm{OII}\right] \lambda 4363$ is not surprising because, of the lines commonly analyzed in the literature, it is these two that deviate from a correlation of critical density with ionization potential.

Note that the velocity shift from systemic is very large $\left(\approx 400 \mathrm{~km} \mathrm{~s}^{-1}\right)$ for the forbidden Fe lines in NGC 5548. The shift is large enough that one may suspect an error in the wavelength calibration. This is not the case, however, because the wavelength calibration matches the known wavelengths of the night-sky lines to a precision of $25 \mathrm{~km}$ $\mathrm{s}^{-1}$. One might also speculate that the $21 \mathrm{~cm}$ systemic velocity measurement is not representative of the NLR center-of mass velocity. However, the $21 \mathrm{~cm}$ systemic velocity agrees (within $40 \mathrm{~km} \mathrm{~s}^{-1}$ ) with $V(50 \%)$ of the lowest critical density lines. Regardless of the systemic velocity, the Fe lines are blueshifted by about $400 \mathrm{~km} \mathrm{~s}^{-1}$ relative to the lowest critical density lines. Thus, NGC 5548 is also unusual in its large blueshift in $V(50 \%)$ of the Fe lines.

In Figures $13 c$ and $13 d$, we plot IPV $(20 \%)$ versus, $n_{\mathrm{cr}}$ and IP. Again, $\left[\mathrm{O}_{1}{ }_{1}\right] \lambda 6300$ deviates from the otherwise weildefined correlation on the IP plot but does not deviate on the $n_{\mathrm{cr}}$ plot. The above correlations for NGC 5548 are consistent with velocity decreasing outward because lines of high critical density are systematically broader than lines of low critical density. In our previous discussion, we have assumed that all Seyfert NLRs are fundamentally similar and can be described by models differing only in, e.g., $N_{\text {col }} / N_{\min }$ at $v_{\min }$. Although the line profiles (especially that of $[\mathrm{Fe} \mathrm{x}]$ 26374) are similar to the profiles of the other objects in our sample, the correlations suggest an NLR with fundamentally different kinematics. In MC, we suggested that at $5 \AA$ and $10 \AA$ resolution, $\lambda 4363$ line widths may have been systematically overestimated in Seyfert 1 galaxies because of difficulties in deblending from the broad and narrow components of $\mathrm{H} \gamma$. Even with our higher resolution, we may be subject to the same effect as well. However, the 
dilemma in which line widths of NGC 5548 seem to correlate fundamentally with critical density rather than with ionization parameter is posed by both $\lambda 4363$ and $\lambda 6300$. The (highly significant) values of $R / P_{\text {null }}$ are $-0.94 / 5.0 \times 10^{-6}$, $-0.83 / 9.2 \times 10^{-4}, 0.88 / 1.8 \times 10^{-4}$, and $0.71 / 9.7 \times 10^{-3}$.

\section{SUMMARY}

The most important observational feature of the profiles presented here is that for lines encompassing a wide range of critical density and ionization potential, the variation of flux with projected velocity is very similar in some objects (e.g., MCG 8-11-11 and NGC 4151). This is inconsistent with a scenario in which lines of low and high critical density and/or ionization potential are formed in physically distinct regions. Thus, we expect comparable spatial extents for emission into each line. We have proposed testing this hypothesis with coordinated Hubble Space Telescope and ground-based spectrophotometry of the applicable NLRs that can be spatially resolved on an angular scale $\left(\$ 2^{\prime \prime}\right)$ that properly complements analyses of existing spatially integrated emission-line spectra. In some other objects, lines of high critical density and/or ionization potential are systematically broader than those of low critical density and/or ionization potential (e.g., Mrk 79, Mrk 704, and Mrk 841). It is possible to explain the difference between the two types of objects in terms of a relatively small difference in the cloud column density throughout the NLR (see Paper II, MC, and Moore [1994]). If clouds at small $r$ have $N_{\mathrm{col}} \approx$ $10^{24} \mathrm{~cm}^{-2}$, then even with $N_{\text {col }} \propto r^{-4 / 3}$ (constant mass spherical clouds with $n_{e} \propto r^{-2}$ ), it is possible to have $N_{\text {col }}>$ $N_{\min }\left(\approx 10^{22} \mathrm{~cm}^{-2}\right.$ for $\left.\Gamma=0.3\right)$ throughout the NLR so that each cloud emits both [O I] and [Fe VII]. If clouds start out with $N_{\text {col }}$ less by a modest factor, then $N_{\text {col }}$ falls to less than $N_{\min }$ before clouds reach their maximum velocity at large $r$, and an [O I] profile narrower than that of [Fe vII] results.
Overall, our sample of Seyfert 1 galaxies confirms the well-known correlations of line width and blueshift with ionization potential and/or critical density. A sample the size of ours cannot alone confirm or refute properly the conclusion of $\mathrm{MC}$ that the correlations are fundamentally with ionization potential rather than with critical density, especially given that our sample includes one unusual counter example (NGC 5548). The correlations for Mrk 841 are not conclusive because the only lines that stand out are [Fe vII] $\lambda 6087$ and $[\mathrm{Fe} \mathrm{x}] \lambda 6374$, which have both high critical density and high ionization potential. This leaves just four objects, MCG 8-11-11, Mrk 79, Mrk 704, and NGC 4151, to support our earlier conclusion (correlations with ionization potential). Nevertheless, these four objects do follow the trend discussed in MC; the objects with broad [O I] profiles (MCG 8-11-11 and NGC 4151) also have broad [S II] profiles, and the objects with narrow [O I] profiles (Mrk 79 and Mrk 704) have narrow [S II] profiles.

We thank Pam Capodicci for very helpful administrative assistance and also thank Ron Lyons for generous technical assistance in preparing the plots. We thank Mark Whittle and the MNRAS for permission to include Figure 7, which was reproduced from Whittle (1985a). We also acknowledge useful discussions with V. T. Junkkarinen, R. C. Puetter, and H. E. Smith. Lastly, we thank our referee, who remained anonymous, for a thoughtful review and for offering suggestions that have improved this paper. This paper is based on observations obtained at the Palomar Observatory while jointly operated by the California Institute of Technology and the Carnegie Observatories. We thank the staff at Mount Palomar for its assistance with the observations. This work has been supported by NASA grant NAG 5-1630.

\section{REFERENCES}

Appenzeller, I., \& Östreicher, R. 1988, AJ, 95, 45

Boksenberg, A., Shortridge, K., Allen, D. A.,, Fosbury, R. A. E., Penston, M. V., \& Savage, A. 1975, MNRAS, 173, 381

Capriotti, E., Foltz, C., \& Byard, P. 1981, ApJ, 245, 396

Cohen, R. D. 1983, ApJ, 273, 489

Dahari, O., \& De Robertis, M. M. 1988, ApJS, 67, 249

De Robertis, M. M., \& Osterbrock, D. E. 1984, ApJ, 286, 171 -1986, ApJ, 301, 727

De Robertis, M. M., \& Shaw, R. A. 1990, ApJ, 348, 421

Heckman, T. M., Balick, B., \& Sullivan, W.T. III. 1978, ApJ, 224, 745

Heckman, T. M., Miley, G. K., van Breugel, W. J. M., \& Butcher, H. R. 1981, ApJ, 247, 403

Komossa, S., \& Schulz, H. 1994, in STSci Symp. Ser., 8 The Analysis of

Emission Lines, ed. R. E. Williams \& M. Livio (Baltimore: STScI), 30

Krinsky, I. S., \& Puetter, R. C. 1992, ApJ, 394, 472

Moore, D. 1994, Ph.D. thesis Univ. California, San Diego . 1995, in preparation
Moore, D., \& Cohen, R. D. 1994, ApJ, 433, 602 (MC)

1996, ApJ, 470, 301 (Paper II)

Oke, J. B., \& Gunn, J. E. 1982, PASP, 94, 586

Osterbrock, D. E. 1981, ApJ, 246, 696

Penston, M. V., Fosbury, R. A. E., Boksenberg, A., Ward, M. J., \& Wilson, A. S. 1984, MNRAS, 208, 347

Tsvetanov, Z. I., Kriss, G. A., Evans, I. N., \& Ford, H. C. 1994, BAAS, 26, 966

Veilleux, S. 1988, AJ, 95, 1695

1991a, ApJS, 75, 357

1991 b ApJ, 369, 331

Whittle, M. 1982, Ph.D. thesis, Cambridge Univ. 1985a, MNRAS, 213,1

1985b, MNRAS, 216, 817

. 1991, in Testing the AGN Paradigm, ed. S. S. Holt, S. G. Neff, \& M. C. Urry (New York: AIP), 607 